\title{
Simultaneous Chemical Process Synthesis and Heat Integration with Unclassified Hot/Cold Process Streams
}

\author{
Lingxun Kong, Venkatachalam Avadiappan, Kefeng Huang, and Christos T. Maravelias
}

\begin{abstract}
We propose a mixed-integer nonlinear programing (MINLP) model for the simultaneous chemical process synthesis and heat integration with unclassified process streams. The model accounts for (1) streams that cannot be classified as hot or cold, and (2) variable stream temperatures and flow rates, thereby facilitating integration with a process synthesis model. The hot/cold stream "identities" are represented by classification binary variables which are (de)activated based on the relative stream inlet and outlet temperatures. Variables including stream temperatures and heat loads are disaggregated into hot and cold variables, and each variable is (de)activated by the corresponding classification binary variable. Stream inlet/outlet temperatures are positioned onto "dynamic" temperature intervals so that heat loads at each interval can be properly calculated. The proposed model is applied to two illustrative examples with variable stream flow rates and temperatures, and is integrated with a superstructure-based process synthesis model to illustrate its applicability.
\end{abstract}

Keywords: Mixed-integer nonlinear programming; superstructure optimization.

\section{Introduction}

Energy consumption has become a critical concern in the chemical process industries due to various economic and environmental reasons. The heat integration between hot and cold streams reduces energy consumption thereby improving energy efficiency. Heat integration has received considerable attention and significant progress has been made over the past decades. Since the concept of heat integration was first introduced by Linnhoff and Flower (1978) upon Hohmann's PhD thesis (Hohmann, 1971), various approaches have been proposed, including the pinch design method by Linnhoff and Hindmarsh (1983), the transportation formulation by Cerda et al. (1983), the transshipment model by Papoulias and Grossmann (1983), the one-stage superstructure based approach by Floudas and Ciric (1991), and the stagewise superstructure based model from Yee and Grossmann (1990). Gundersen and Naess (1988), Jeżowski (1994), Furman and Sahinidis (2001), Morar and Agachi (2010), and Klemeš and Kravanja (2013) have reviewed extensively the literature on heat integration. The aforementioned approaches are sequential: the chemical process is first optimized, and heat integration is performed with fixed stream conditions (e.g. temperatures and 
flow rates) to minimize utility consumption. These sequential strategies do not account for the strong interactions between the chemical process and heat integration subsystems.

On the other hand, the simultaneous design of the chemical process and the heat exchanger network optimizes the overall process. Papoulias and Grossmann (1983) first introduced a strategy based on mixed-integer linear programming (MILP) to predict the minimum utility consumption with variable process stream flow rates. However, the stream temperatures in this model have to be prespecified. To overcome this limitation, Duran and Grossmann (1986) used a nonlinear programming (NLP) model with a set of inequality constraints to model the "max" functions that identify the pinch temperature for variable process stream temperatures and flow rates. This model has been used as a basis for subsequent work on process synthesis (Dowling \& Biegler, 2015; El - Halwagi \& Manousiouthakis, 1990; Kamath, Biegler, \& Grossmann, 2012; Pattison \& Baldea, 2015; Srinivas \& ElHalwagi, 1994; Yuan \& Eden, 2016). While the "max" function can be approximated (Balakrishna \& Biegler, 1992), the selection of the parameters for the continuously differentiable function can be non-trivial. To avoid the non-differentiability in the model of Duran and Grossmann (1986), subsequent approaches employed binary variables to locate pinch temperatures. For instance, Grossmann et al. (1998) presented a disjunctive model to explicitly locate a stream above, across or below a potential pinch candidate. Navarro-Amorós et al. (2013) presented an alternative disjunctive model that uses temperature intervals and the transshipment model for heat integration with variable temperatures. Hui (2014) proposed a model that utilizes pseudo stream temperatures. Tan et al. (2014) also used binary variables to parameterize the stream locations but introduced constraints with the products of a binary and a continuous variable, which led to Big-M formulations after linearization.

One basic assumption in all previous approaches is that we are given a set of hot streams to be cooled and a set of cold streams to be heated. However, in many cases, especially in the context of superstructure optimization, some process streams cannot be classified a priori as hot or cold streams. To illustrate, consider the outlet stream from one of two alternative reactors (A or B) which is sent to one of the two alternative separation units ( $C$ or D) for purification, where units $A, B, C$ and D operate at different temperatures. As shown in Figure 1, we cannot determine if the stream that connects the reactor and the separator is a hot or cold stream before solving the model.

Accordingly, in this paper we present a general heat integration model that not only handles variable stream temperatures and flow rates, but also handles unclassified hot/cold streams. The rest of the paper is structured as follows. In Section 2, we present the proposed MINLP formulation. In Section 3 , we present two extensions, and in Section 4, we show how our model can be modified to handle phase changes. In Section 5, we discuss solution methods. In Section 6, we show how the proposed model can be used to address process synthesis problems and in Section 7, we provide two illustrative examples along with a large example in which the proposed model is used for the design of a chemical process using superstructure optimization. 


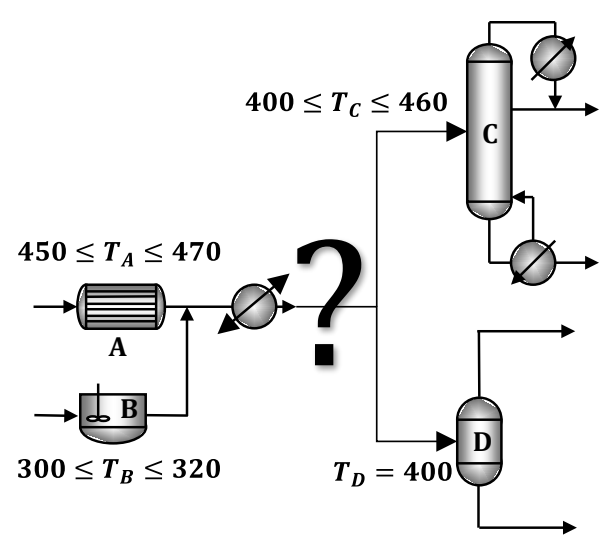

Figure 1. An example of an unclassified hot/cold process stream in a process superstructure. Only one reactor and one separator can be selected in any feasible solution.

\section{Heat Integration Model}

The proposed heat integration model is based upon the linear programing (LP) transshipment model proposed by Papoulias and Grossmann (1983). The LP transshipment model utilizes the concept of temperature intervals to predict the minimum utility consumption for a process with fixed stream conditions. Under the assumption of constant heat capacity of process streams, the temperature intervals are constructed from the known inlet temperatures of the streams and utilities. Hot streams and utilities are treated as source nodes, while cold streams and utilities are considered sink nodes. Heat is transferred like a "commodity" between sources and sinks via temperature intervals, which can be seen as intermediate "warehouses". Any residue heat is cascaded down to the next interval at a lower temperature. Heat integration using this approach maximizes heat exchange among streams, while satisfies the second law of thermodynamics and minimum approach temperature as a heat exchanger design requirement. As a result of the fixed stream temperatures and flow rates, the only variables in this LP model are the residue heat at each interval and the heat duties of hot and cold utilities.

To account for variable stream temperatures and flow rates, Navarro et. al. extended the LP transshipment model and proposed a disjunctive model. In their work, "dynamic" temperature intervals are constructed through an implicit ordering of stream inlet temperatures. Extending and generalizing this concept, we propose a model that not only allows variable stream conditions, but also handles streams that cannot be classified as hot or cold a priori.

Given a process stream with fixed composition, the heat capacity can be assumed constant if it does not vary significantly with temperature. When the temperature dependence has to be taken into account, the stream can be divided into sub-streams, each with constant heat capacity, so that a piecewise linear approximation of the temperature-enthalpy $(\mathrm{T}-\mathrm{H})$ relation is obtained. If a stream composition is not fixed, then the stream or sub-stream heat capacity can be defined as a function (variable) of composition. Thus, all cases of variable heat capacities can be addressed, albeit at the expense of computational performance. 


\subsection{Classification of Hot/Cold Streams}

The hot/cold identity of an unclassified process stream can be determined by comparing the stream inlet $\left(\right.$ TIN $_{S}$ ) and outlet (TOUT $T_{S}$ ) temperatures; e.g., if a stream has a higher temperature at its inlet than at its outlet, it is a hot stream. The comparison of temperatures is carried out using a pair of classification binary variables, $W_{s}^{H}$ and $W_{s}^{C}$, as shown in Eqs. (1) - (4). If process stream $s \in \mathbf{S}^{\mathbf{P}}$ is a hot stream, $W_{s}^{H}=1$ and $W_{s}^{C}=0$, while if it is a cold stream, $W_{s}^{C}=1$ and $W_{s}^{H}=0$ :

$W_{s}^{H}+W_{s}^{C}=1$

$s \in \mathbf{S}^{\mathbf{P}}$

$\operatorname{TIN}_{S}-$ TOUT $_{S}=T_{S}^{+}-T_{S}^{-}$

$s \in \mathbf{S}^{\mathbf{P}}$

$T_{s}^{+} \leq \alpha_{s}^{U} W_{s}^{H}$

$s \in \mathbf{S}^{\mathbf{P}}$

$T_{s}^{-} \leq \alpha_{s}^{U} W_{s}^{C}$

$s \in \mathbf{S}^{\mathbf{P}}$

where $\alpha_{s}^{U}$ are valid upper-bounds on the absolute differences between stream inlet and outlet temperatures. Eq. (1) ensures that each stream is classified either as hot or cold. Non-negative variables $T_{s}^{+}$and $T_{s}^{-}$are used in Eq. (2) to model the temperature difference between the inlet and outlet of each process stream, and they are (de)activated by the corresponding classification binaries in Eqs. (3) and (4).

Next, variables $\left(x_{s}\right)$ related to an unclassified stream are disaggregated into two counter parts, $x_{s}^{H}$ and $x_{s}^{C}$. If the stream is classified to be a hot stream, then we enforce that $x_{s}=x_{s}^{H}$ and $x_{s}^{C}=0$, while if it is a cold stream we enforce $x_{s}=x_{s}^{C}$ and $x_{s}^{H}=0$. In this way we can formulate constraints for the "hot" and "cold" parts separately (Lee \& Grossmann, 2005; Türkay \& Grossmann, 1996).

$\left[\begin{array}{c}W_{s}^{H} \\ \neg W_{s}^{C} \\ x_{s}=x_{s}^{H}\end{array}\right] \underline{\vee}\left[\begin{array}{c}\neg W_{s}^{H} \\ W_{s}^{C} \\ x_{s}=x_{s}^{C}\end{array}\right]$

$s \in \mathbf{S}^{\mathbf{P}}$

The linear reformulation of the above disjunctions will be discussed in the next subsections. For consistency, throughout the paper, superscript $\mathrm{H} / \mathrm{C}$ is used to denote disaggregated variables for hot/cold streams.

\subsection{Dynamic Interval Construction}

In the proposed model, temperature intervals cannot be directly constructed since the stream inlet (and outlet) temperatures are not known prior to solving the model. To address this, we construct "dynamic" intervals in which the variable stream inlet temperatures are ordered from high to low. In general, the minimum number of interval boundaries (i.e. number of intervals plus one) required should be the same as the number of streams including process streams and utilities. Here we use set $\mathbf{K}$ to denote interval boundaries and $\mathbf{K}^{\mathbf{I}}=\mathbf{K} \backslash\{0\}$ to denote temperature intervals. We introduce nonnegative variables $T_{k}$ to denote the temperature at the interval boundaries, ordered as follows:

$T_{k} \leq T_{k-1}$

$k \in \mathbf{K}^{\mathbf{I}}$

Since interval boundaries are defined by stream inlet temperatures, each boundary temperature, $T_{k}$, corresponds to one distinct inlet temperature $\left(\operatorname{TIN}_{S}\right)$. 
This one-to-one matching can be done using a pair of binary variables, $X_{s, k}^{H}$ and $X_{s, k}^{C}$, which are equal to 1 only when $T_{I} N_{s}$ is equal to $T_{k}$. We first enforce that each stream inlet temperature must be assigned to one interval boundary:

$\begin{array}{ll}\sum_{k} X_{s, k}^{H}=W_{s}^{H} & s \in \mathbf{S}^{\mathbf{P}} \\ \sum_{k} X_{s, k}^{C}=W_{s}^{C} & s \in \mathbf{S}^{\mathbf{P}}\end{array}$

Depending on the hot/cold identity of each process stream, we allow only one $X_{s, k}^{H}$ or one $X_{s, k}^{C}$ to be equal to 1 at any interval. The above constraints connect $X_{s, k}^{H}$ and $X_{s, k}^{C}$ to the classification binaries so that the disjunction in Eq. (5) is enforced.

Then, through Eq. (8) it is ensured that only one stream has its inlet temperature assigned to each boundary.

$\sum_{s \in \mathbf{S}}\left(X_{s, k}^{H}+X_{s, k}^{C}\right)=1 \quad k \in \mathbf{K}$

Finally, we disaggregate the inlet temperatures into hot/cold parts as well as into temperature intervals (i.e. $T I N_{s, k}^{H}$ and $T I N_{S, k}^{C}$ ) via Eq. (9).

$\operatorname{TIN}_{S}=\sum_{k \in \mathbf{K}}\left(\operatorname{TIN}_{s, k}^{H}+\operatorname{TIN}_{s, k}^{C}\right) \quad s \in \mathbf{S}$

These disaggregated inlet temperatures should be equal to the boundary temperatures only when the corresponding $X_{S, k}^{H}$ or $X_{s, k}^{C}$ is equal to one, while they are forced to zero otherwise,

$\begin{array}{ll}\operatorname{TIN}_{s, k}^{H} \leq \beta_{s}^{U} X_{s, k}^{H} & s \in \mathbf{S}, k \in \mathbf{K} \\ \operatorname{TIN}_{s, k}^{H} \leq T_{k} & s \in \mathbf{S}, k \in \mathbf{K} \\ \operatorname{TIN}_{s, k}^{H} \geq T_{k}-\beta_{s}^{U}\left(1-X_{s, k}^{H}\right) & s \in \mathbf{S}, k \in \mathbf{K} \\ \operatorname{TIN}_{s, k}^{C} \leq \beta_{s}^{U} X_{s, k}^{C} & s \in \mathbf{S}, k \in \mathbf{K} \\ \operatorname{TIN}_{s, k}^{C} \leq T_{k}-\delta & s \in \mathbf{S}, k \in \mathbf{K} \\ \operatorname{TIN}_{S, k}^{C} \geq\left(T_{k}-\delta\right)-\left(\beta_{s}^{U}-\delta\right)\left(1-X_{s, k}^{C}\right) & s \in \mathbf{S}, k \in \mathbf{K}\end{array}$

where $\beta_{s}^{U}$ are valid upper bounds on the stream inlet temperatures. If a process stream is hot $\left(W_{s}^{H}=\right.$ $\left.1, W_{s}^{C}=0\right)$, then Eq. $(7 \mathrm{H})$ enforces exactly one $X_{s, k}^{H}$ to be equal to one, while Eq. (7C) forces all the $X_{s, k}^{C}$ to zero. Disaggregated variables $T I N_{s, k}^{H}$ and $T I N_{s, k}^{C}$ are (de)activated as follows: Eq. (10H) forces $\operatorname{TIN}_{s, k}^{H}$ to zero when the corresponding $X_{s, k}^{H}$ is equal to zero; Eqs. $(11 \mathrm{H})$ and $(12 \mathrm{H})$ enforce that $\operatorname{TIN}_{s, k}^{H}=T_{k}$ only when $X_{s, k}^{H}=1$; Eq. (10C) enforces that all $T I N_{s, k}^{C}$ to be equal to zero; and Eqs. (11C) and $(12 \mathrm{C})$ are not active. Finally, the non-zero $T I N_{S, k}^{H}$ connects the stream inlet temperature $\left(T I N_{S}\right)$ and the boundary temperature $\left(T_{k}\right)$ via Eq. (9). Note that all the cold part of the disaggregated inlet temperatures are shifted by the heat recovery approach temperature $(\delta)$ as a heat exchanger design requirement.

\subsection{Outlet Temperature Assignment}

While the stream outlet temperatures $\left(\mathrm{TOUT}_{s}\right)$ are not used to construct the temperature intervals, they must be correctly positioned with respect to the interval boundaries in order to calculate heat 
duties at each interval. Therefore, we introduce another pair of binary variables $\left(Y_{s, k}^{H} / Y_{s, k}^{C}\right)$, which are equal to one if the outlet temperature is within interval $k$.

First, we enforce that each stream outlet temperature is located at exactly one interval:

$\sum_{k \in \mathbf{K}^{\mathbf{I}}} Y_{s, k}^{H}=W_{s}^{H} \quad s \in \mathbf{S}^{\mathbf{P}}$

$\sum_{k \in \mathbf{K}^{\mathrm{I}}} Y_{s, k}^{C}=W_{s}^{C} \quad s \in \mathbf{S}^{\mathbf{P}}$

Then, similarly to the previous section, the stream outlet temperatures are disaggregated into $\operatorname{TOUT}_{s, k}^{H} / \operatorname{TOUT}_{s, k}^{C}$, according to the corresponding $Y_{s, k}^{H} / Y_{s, k}^{C}$. If $Y_{s, k}^{H}=1$, then $\operatorname{TOUT}_{s, k}^{H} \in\left[T_{k}, T_{k-1}\right]$; if $Y_{S, k}^{C}=1$, then $\operatorname{TOUT}_{s, k}^{C} \in\left[T_{k}-\delta, T_{k-1}-\delta\right]$; while if $Y_{s, k}^{H} / Y_{s, k}^{C}$ are zero, the corresponding $T O U T_{s, k}^{H} /$ $\operatorname{TOUT}_{S, k}^{C}$ must be zero,

$\operatorname{TOUT}_{s}=\sum_{k \in \mathbf{K}^{\mathrm{I}}}\left(\right.$ TOUT $_{s, k}^{H}+$ TOUT $\left._{S, k}^{C}\right) \quad s \in \mathbf{S}^{\mathbf{P}}$

$\operatorname{TOUT}_{S, k}^{H} \leq \gamma_{S}^{U} Y_{s, k}^{H}$

$s \in \mathbf{S}^{\mathbf{P}}, k \in \mathbf{K}^{\mathbf{I}}$

$\operatorname{TOUT}_{s, k}^{H} \leq T_{k-1}$

$s \in \mathbf{S}^{\mathbf{P}}, k \in \mathbf{K}^{\mathbf{I}}$

$\operatorname{TOUT}_{s, k}^{H} \geq T_{k}-\gamma_{s}^{U}\left(1-Y_{s, k}^{H}\right)$

$s \in \mathbf{S}^{\mathbf{P}}, k \in \mathbf{K}^{\mathbf{I}}$

$\operatorname{TOUT}_{S, k}^{C} \leq \gamma_{S}^{U} Y_{s, k}^{C}$

$s \in \mathbf{S}^{\mathbf{P}}, k \in \mathbf{K}^{\mathbf{I}}$

$\operatorname{TOUT}_{s, k}^{C} \leq T_{k-1}-\delta$

$s \in \mathbf{S}^{\mathbf{P}}, k \in \mathbf{K}^{\mathbf{I}}$

$\operatorname{TOUT}_{S, k}^{C} \geq\left(T_{k}-\delta\right)-\left(\gamma_{S}^{U}-\delta\right)\left(1-Y_{S, k}^{C}\right)$

$s \in \mathbf{S}^{\mathbf{P}}, k \in \mathbf{K}^{\mathbf{I}}$

where $\gamma_{s}^{U}$ are valid upper bounds on the stream outlet temperatures. Again, Eq. (14) disaggregates the outlet temperatures, and Eq. $(15 \mathrm{H})$ and $(15 \mathrm{C})$ force the disaggregated temperatures to zero when the corresponding binaries are equal to zero. The main difference compared to the "inlet" counterpart is that equality is not enforced here when $Y_{s, k}^{H} / Y_{s, k}^{C}$ is equal to one (see Eqs. $(16 \mathrm{H})-(17 \mathrm{H})$ and (16C) - (17C)). Figure 2 provides an example of the assignment of stream inlet and outlet temperatures to temperature intervals.

\subsection{Heat Duty Calculations}

In order to calculate the heat duty of a given stream $s$ at interval $k$, we have to consider the following three possible cases:

(1) the stream completely spans (i.e. goes through) interval $k$,

(2) the stream partially spans interval $k$, and

(3) the stream does not span any portion of interval $k$. 


\begin{tabular}{|c|c|c|c|c|c|c|c|c|c|}
\hline \multicolumn{4}{|c|}{ Boundaries $k \in \mathbf{K}$} & \multicolumn{3}{|c|}{ Intervals $k \in \mathbf{K}^{\mathbf{I}}$} & \multicolumn{3}{|c|}{ Boundaries $k \in \mathbf{K}$} \\
\hline$T_{k}$ & $X_{1, k}^{H}$ & $\operatorname{TIN}_{1, k}^{H}$ & & r & 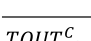 & & $\operatorname{TIN}_{2, k}^{C}$ & $X_{2, k}^{C}$ & $T_{k}^{\prime}$ \\
\hline$T_{0}$ & 1 & $T_{0}$ & ${ }_{1}$, & & 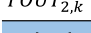 & & 0 & 0 & $T_{0}^{\prime}$ \\
\hline$T_{1}$ & 0 & 0 & 0 & 0 & {$\left[T_{1}^{\prime}, T_{0}^{\prime}\right]$} & 1 & 0 & 0 & $T^{\prime}$ \\
\hline$I_{1}$ & & U & 0 & 0 & 0 & 0 & U & & $I_{1}$ \\
\hline$T_{2}$ & 0 & 0 & 1 & {$\left[T_{3}, T_{2}\right]$} & 0 & 0 & 0 & 0 & $T_{2}^{\prime}$ \\
\hline$T_{3}$ & 0 & 0 & 0 & 0 & 0 & 0 & 0 & 0 & $T_{3}^{\prime}$ \\
\hline$T_{4}$ & 0 & 0 & & & & & $T_{4}^{\prime}$ & 1 & $T_{4}^{\prime}$ \\
\hline
\end{tabular}

Figure 2. Temperature assignment of a hot stream (left) and a cold stream (right) to temperature intervals. The hot stream starts at interval boundary 0 and ends at interval 3, while the cold stream starts at interval boundary 4 and ends at interval 1 . Also note that $T_{k}^{\prime}=T_{k}-\delta$.

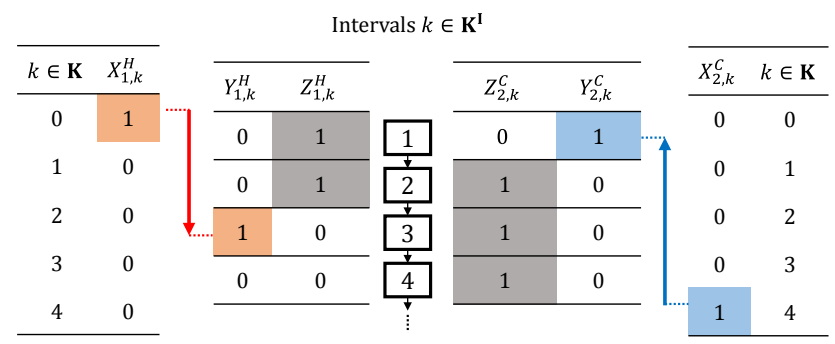

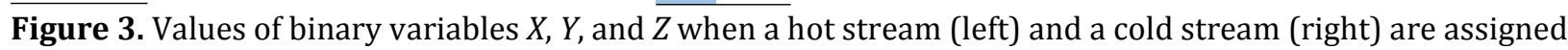
to the temperature intervals.

Case (2) is true when $Y_{s, k}^{H} / Y_{s, k}^{C}=1$, whereas to represent cases (1) and (3) we need additional binary variables. We introduce binary variables $Z_{s, k}^{H} / Z_{s, k}^{C}$, which are equal to one when stream $s$ goes through interval $k$ (i.e. case (1) is true). The constraints that relate $Z_{s, k}^{H} / Z_{s, k}^{C}$ with binary variables $X$ and $Y$ are as follows,

$Z_{s, k}^{H}=Z_{s, k-1}^{H}+X_{s, k-1}^{H}-Y_{s, k}^{H}$

$$
\begin{aligned}
& s \in \mathbf{S}^{\mathbf{P}}, k \in \mathbf{K}^{\mathbf{I}} \\
& s \in \mathbf{S}^{\mathbf{P}}, k \in \mathbf{K}^{\mathbf{I}}
\end{aligned}
$$$$
Z_{s, k-1}^{C}=Z_{s, k}^{C}+X_{s, k-1}^{C}-Y_{s, k-1}^{C}
$$

They ensure that if a stream starts at boundary $k^{\prime} \in \mathbf{K}$ and ends somewhere within interval $k^{\prime \prime} \in \mathbf{K}^{\mathbf{I}}$, then it must have gone through all the intervals from $k^{\prime}+1$ to $k^{\prime \prime}-1$ for hot stream and from $k^{\prime \prime}+$ 1 to $k^{\prime}$ for cold stream. The logic is illustrated in Figure 3.

Thus, when $Z_{s, k}^{H} / Z_{s, k}^{C}$ are equal to one, case (1) is true, while when neither $Y_{s, k}^{H} / Y_{s, k}^{C}$ nor $Z_{s, k}^{H} / Z_{s, k}^{C}$ are equal to one, case (3) is true. The heat duty of a stream at interval $k$ is disaggregated into $Q_{s, k}^{H}$ and $Q_{s, k}^{C}$, and $Q_{s, k}^{H} / Q_{s, k}^{C}$ are further disaggregated into two mutually exclusive counterparts: $Q 1_{s, k}^{H} / Q 1_{s, k}^{C}$ representing heat duties in case (1), and $Q 2_{s, k}^{H} / Q 2_{s, k}^{C}$ representing those in case (2). Note that the heat duties in case (3) should always be zero and are therefore not considered in the following constraints:

$$
\begin{array}{lc}
Q Q_{s, k}^{H}=Q 1_{s, k}^{H}+Q 2_{s, k}^{H} & s \in \mathbf{S}^{\mathbf{P}}, k \in \mathbf{K}^{\mathbf{I}} \\
Q 1_{s, k}^{H} \leq \theta 1_{s}^{U} Z_{s, k}^{H} & s \in \mathbf{S}^{\mathbf{P}}, k \in \mathbf{K}^{\mathbf{I}} \\
Q 2_{s, k}^{H} \leq \theta 2_{s}^{U} Y_{s, k}^{H} & s \in \mathbf{S}^{\mathbf{P}}, k \in \mathbf{K}^{\mathbf{I}} \\
Q Q_{s, k}^{C}=Q 1_{s, k}^{C}+Q 2_{s, k}^{C} & s \in \mathbf{S}^{\mathbf{P}}, k \in \mathbf{K}^{\mathbf{I}} \\
Q 1_{s, k}^{C} \leq \theta 1_{s}^{U} Z_{s, k}^{C} & s \in \mathbf{S}^{\mathbf{P}}, k \in \mathbf{K}^{\mathbf{I}}
\end{array}
$$


$Q 2_{s, k}^{C} \leq \theta 2_{s}^{U} Y_{s, k}^{C}$

$s \in \mathbf{S}^{\mathbf{P}}, k \in \mathbf{K}^{\mathbf{I}}$

For illustration, let us assume we are given a hot stream $s$ at interval $k$. Eqs. (19C) - (21C) ensure that $Q_{s, k}^{C}, Q 1_{s, k}^{C}$, and $Q 2_{s, k}^{C}$ are zero when $s$ is a hot stream. If the stream goes through interval $k$ (case (1)), Eqs. (19H) - (21H) ensure that $Q_{s, k}^{H}=Q 1_{s, k}^{H}$; if it partially spans the interval (case (2)), $Q_{s, k}^{H}=$ $Q 2_{s, k}^{H}$ is enforced; and if it does not span any portion of the interval (case (3)), we obtain $Q_{s, k}^{H}=0$.

The next step is to relate the heat duties to the temperature differences and heat capacity flows $\left(F_{s}\right)$. For simplicity, we first assume that $s$ is a hot stream $\left(Q_{s, k}^{C}=Q 1_{s, k}^{C}=Q 2_{s, k}^{C}=0\right)$. The heat duties in case (1), $Q 1_{s, k}^{H}$, can be determined as follows,

$Q 1_{s, k}^{H}+Q 1_{s, k}^{H, D}=F_{S}\left(T_{k-1}-T_{k}\right)$

$s \in \mathbf{S}^{\mathbf{P}}, k \in \mathbf{K}^{\mathbf{I}}$

$Q 1_{s, k}^{H, D} \leq \theta 1_{s}^{U}\left(1-Z_{s, k}^{H}\right)$

$s \in \mathbf{S}^{\mathbf{P}}, k \in \mathbf{K}^{\mathbf{I}}$

where $Q 1_{s, k}^{H, D}$ is a nonnegative slack variable that takes the value of the right-hand-side (RHS) of Eq. $(22 \mathrm{H})$ if case (1) is not true (i.e. $Z_{s, k}^{H}=0$ ). If case (1) is true, $Q 1_{s, k}^{H}$ is equal to the heat capacity flow multiplied by the temperature difference between the interval boundaries.

Similarly, the heat duty in case (2), $Q 2_{s, k}^{H}$, is calculated as follows,

$$
\begin{array}{ll}
Q 2_{s, k}^{H}+Q 2_{s, k}^{H, D}=F_{s}\left(T_{k-1}-\operatorname{TOUT}_{s, k}^{H}\right) & s \in \mathbf{S}^{\mathbf{P}}, k \in \mathbf{K}^{\mathbf{I}} \\
Q 2_{s, k}^{H, D} \leq \theta 2_{s}^{U}\left(1-Y_{s, k}^{H}\right) & s \in \mathbf{S}^{\mathbf{P}}, k \in \mathbf{K}^{\mathbf{I}}
\end{array}
$$

where $Q 2_{s, k}^{H, D}$ is a nonnegative slack variable that is equal to the RHS of Eq. $(24 \mathrm{H})$ if case (2) is not true (i.e. $Y_{s, k}^{H}=0$ ). When case (2) is true, $Q 2_{s, k}^{H, D}$ is equal to zero and the heat duty is determined from the stream heat capacity flow and the difference between the outlet temperature and boundary temperature.

Similar constraints are introduced for the cold streams:

$$
\begin{array}{lc}
Q 1_{s, k}^{C}+Q 1_{s, k}^{C, D}=F_{s}\left(T_{k-1}-T_{k}\right) & s \in \mathbf{S}^{\mathbf{P}}, k \in \mathbf{K}^{\mathbf{I}} \\
Q 1_{s, k}^{C, D} \leq \theta 1_{s}^{U}\left(1-Z_{s, k}^{C}\right) & s \in \mathbf{S}^{\mathbf{P}}, k \in \mathbf{K}^{\mathbf{I}} \\
Q 2_{s, k}^{C}+Q 2_{s, k}^{C, D}-Q 2_{s, k}^{C, P}=F_{s}\left(T O U T_{s, k}^{C}-T_{k}+\delta\right) & s \in \mathbf{S}^{\mathbf{P}}, k \in \mathbf{K}^{\mathbf{I}} \\
Q 2_{s, k}^{C, D} \leq \theta 2_{s}^{U}\left(1-Y_{s, k}^{C}\right) & s \in \mathbf{S}^{\mathbf{P}}, k \in \mathbf{K}^{\mathbf{I}} \\
Q 2_{s, k}^{C, P} \leq \theta 2_{s}^{U}\left(1-Y_{s, k}^{C}\right) & s \in \mathbf{S}^{\mathbf{P}}, k \in \mathbf{K}^{\mathbf{I}}
\end{array}
$$

where $Q 1_{s, k}^{C, D}$ and $Q 2_{s, k}^{C, D}$ are the cold counter part of $Q 1_{s, k}^{H, D}$ and $Q 2_{s, k}^{H, D}$, respectively. The reason we need a pair of slack variables $\left(Q 2_{s, k}^{C, D}\right.$ and $\left.Q 2_{s, k}^{C, P}\right)$ for cold streams is that the RHS of Eq. (24C) can be either positive or negative depending on the sign of $\left(\operatorname{TOUT}_{s, k}^{C}-T_{k}+\delta\right)$. The heat duty calculation is further illustrated using an example in Figure 4.

For now, we assume a single isothermal hot utility at the highest temperature interval, and a single cold utility that enters and exits at the lowest interval. Therefore, constraints in Section 2.4 are only written for the process streams $\left(s \in \mathbf{S}^{\mathbf{P}}\right)$. In Section 3, we will extend our discussion on how to calculate heat duties if multiple utility streams are present. 


\subsection{Generalized Transshipment Model}

Using $Q_{s, k}^{H}$ and $Q_{S, k}^{C}$ from the previous section, we write the heat flow balance at interval $k$ :

$R_{k}-R_{k-1}=\sum_{s \in \mathbf{S}}\left(Q_{s, k}^{H}-Q_{s, k}^{C}\right)$

$k \in \mathbf{K}^{\mathbf{I}}$

where $R_{k-1}$ and $R_{k}$ are residue heats entering and exiting interval $k$, respectively. We enforce $R_{0}=$ $R_{|\mathbf{K}|}=0$ so that no residue heat is added to the highest temperature interval or received from lowest temperature interval.

\begin{tabular}{|c|c|c|c|c|c|}
\hline \multirow[b]{2}{*}{ hot stream, s1 } & \multicolumn{2}{|c|}{$Q 1^{H}$} & \multicolumn{2}{|c|}{$Q 2^{H}$} & \multirow{2}{*}{$Q_{1, k}^{H}=Q 1_{1, k}^{H}+Q 2_{1, k}^{H}$} \\
\hline & $Q 1_{1, k}^{H}$ & $Q 1_{1, k}^{H, D}$ & $Q 2_{1, k}^{H}$ & $Q 2_{1, k}^{H, D}-Q 2_{1, k}^{H, P}$ & \\
\hline$Y_{1,1}^{H}=0, Z_{1,1}^{H}=0$ & 0 & $F_{1}\left(T_{0}-T_{1}\right)$ & 0 & $F_{1}\left(T_{0}-\operatorname{TOUT}_{1,1}^{H}\right)$ & 0 \\
\hline$Y_{1,2}^{H}=0, Z_{1,2}^{H}=1$ & $F_{1}\left(T_{1}-T_{2}\right)$ & 0 & 0 & $F_{1}\left(T_{1}-\operatorname{TOUT}_{1,2}^{H}\right)$ & $F_{1}\left(T_{1}-T_{2}\right)$ \\
\hline$Y_{1,3}^{H}=1, Z_{1,3}^{H}=0$ & 0 & $F_{1}\left(T_{2}-T_{3}\right)$ & $F_{1}\left(T_{2}-\operatorname{TOUT}_{1,3}^{H}\right)$ & 0 & $F_{1}\left(T_{2}-\operatorname{TOUT}_{1,3}^{H}\right)$ \\
\hline
\end{tabular}

\begin{tabular}{|c|c|c|c|c|c|}
\hline \multirow[b]{2}{*}{ cold stream, s2 } & \multicolumn{2}{|r|}{$Q 1^{C}$} & \multicolumn{2}{|c|}{$Q 2^{C}$} & \multirow{2}{*}{$Q_{2, k}^{C}=Q 1_{2, k}^{C}+Q 2_{2, k}^{C}$} \\
\hline & $Q 1_{2, k}^{C}$ & $Q 1_{2, k}^{C, D}$ & $Q 2_{2, k}^{C}$ & $Q 2_{2, k}^{\mathrm{C}, D}-Q 2_{2, k}^{\mathrm{C}, P}$ & \\
\hline$-\delta Y_{Z, \mathbf{1}}^{c}=\mathbf{0}, Z_{2, \mathbf{1}}^{c}=\mathbf{0}$ & 0 & $F_{2}\left(T_{0}-T_{1}\right)$ & 0 & $F_{2}\left(\operatorname{TOUTT}_{2,1}^{C}-T_{1}+\delta\right)$ & 0 \\
\hline$T_{2}-\delta \stackrel{Y_{2,2}^{C}=1, Z_{2,2}^{c}=0}{ }$ & 0 & $F_{2}\left(T_{1}-T_{2}\right)$ & $F_{2}\left(\operatorname{TOUT}_{2,2}^{C}-T_{2}+\delta\right)$ & 0 & $F_{2}\left(\right.$ TOUT T $\left._{2,2}^{C}-T_{2}+\delta\right)$ \\
\hline$T_{3}-\delta \frac{Y_{2,3}^{C_{1}}=0, Z_{2,3}^{C}=0}{C_{1}}$ & 0 & $F_{2}\left(T_{2}-T_{3}\right)$ & 0 & $F_{2}\left(\right.$ TOUT $\left._{2,3}^{C}-T_{3}+\delta\right)$ & 0 \\
\hline
\end{tabular}

Figure 4. Heat duty calculations in the heat cascade for a hot stream (s1) and a cold stream (s2). The last column represents the actual heat duty of the stream at each interval, and the shaded cells represent the slack variables that are not used to calculate the actual heat duty.

For minimum utility targeting, the objective function can be written as follows,

$\min \sum_{s \in \mathbf{S}^{\mathrm{HU}}} \sum_{k \in \mathbf{K}^{\mathbf{I}}} \mu_{s} Q_{s, k}^{H}+\sum_{s \in \mathbf{S}^{\mathrm{CU}}} \sum_{k \in \mathbf{K}^{\mathbf{I}}} \mu_{s} Q_{s, k}^{c}$

where $\mu_{s}$ is the unit cost of each utility stream. This expression can account for multiple utilities.

If we consider the entire chemical facility, the objective function must be modified to include terms representing the capital and operating cost of the process,

$\min \sum_{s \in \mathbf{S}^{\mathrm{HU}}} \sum_{k \in \mathbf{K}^{\mathrm{I}}} \mu_{s} Q_{s, k}^{H}+\sum_{s \in \mathbf{S}^{\mathrm{CU}}} \sum_{k \in \mathbf{K}^{\mathrm{I}}} \mu_{s} Q_{s, k}^{c}+f(\Psi)$

where $\Psi$ is a vector of variables such as flow rates, temperatures, pressures and equipment sizes, and $f(\Psi)$ is a cost function that relates these variables to the annualized costs. This objective is used in the examples presented in Section 7. 


\section{Extensions}

In this section, we discuss how the model can be modified to handle multiple utilities and isothermal streams.

\subsection{Multiple Utilities}

If the utility is an isothermal stream (e.g. medium pressure steam), it exchanges heat with other streams at a constant temperature, which means that it defines an interval boundary on which it exchanges heat with other streams. Therefore, the isothermal utility usage is determined through binary variables $X_{s, k}^{H} / X_{s, k}^{C}$,

$Q_{s, k}^{H} \leq \theta_{s}^{U} X_{s, k}^{H}$

$$
\begin{aligned}
& s \in \mathbf{S}^{\mathbf{I H U}}, k \in \mathbf{K}^{\mathbf{I}} \\
& s \in \mathbf{S}^{\mathbf{I C U}}, k \in \mathbf{K}^{\mathbf{I}}
\end{aligned}
$$$$
Q_{s, k}^{C} \leq \theta_{S}^{U} X_{s, k}^{C}
$$

where $Q_{s, k}^{C}=0$ for isothermal hot utilities $\left(s \in \mathbf{S}^{\mathbf{I H U}}\right)$ and $Q_{s, k}^{H}=0$ for isothermal cold utilities $(s \in$ $\left.S^{\text {ICU }}\right)$.

If the utility is a non-isothermal stream (e.g. hot water) that could span multiple temperature intervals, then, in the general case, is treated as a hot/cold non-isothermal process stream. In other words, we need binary variables $X, Y$, and $Z$, and constraints (7) - (26C) to determine the heat duty of the non-isothermal utility at each interval. Since utility streams can always be defined as hot or cold streams and their inlet and outlet temperatures are usually given, we can use preprocessing to reduce the search space and improve the solution process (see details in Section 5).

\subsection{Isothermal Streams}

So far we have assumed that all process streams have different inlet and outlet temperatures, i.e., we have only considered sensible heat. If we are given an isothermal stream ( $\left.s \in \mathbf{S}^{\mathbf{I P}}\right)$, which exchanges heat through evaporation or condensation (latent heat), we assume that the exchange takes place at the boundary it defines. Thus, similar to the case of isothermal utilities, the amount of heat exchanged is constrained via,

$Q_{s, k}^{H} \leq \theta_{s}^{U} X_{s, k}^{H} \quad s \in \mathbf{S}^{\mathbf{I P}}, k \in \mathbf{K}^{\mathbf{I}}$

$Q_{s, k}^{C} \leq \theta_{s}^{U} X_{s, k}^{C}$

$s \in \mathbf{S}^{\mathbf{I P}}, k \in \mathbf{K}^{\mathbf{I}}$

where the disaggregated heat duties, $Q_{s, k}^{H} / Q_{s, k}^{C}$, are related to stream mass flow rate $\left(\widehat{F}_{s}\right)$ and latent heat $\left(\lambda_{s}\right)$,

$\widehat{F}_{s} \lambda_{s}=\sum_{k}\left(Q_{s, k}^{H}+Q_{s, k}^{C}\right) \quad s \in \mathbf{S}^{\mathbf{I P}}$

If the hot/cold classification of the isothermal stream is unknown, we include a dummy temperature difference between the stream inlet and outlet in order to classify the stream:

TIN $_{s}-$ TOUT $_{s}=W_{s}^{H}-W_{s}^{C} \quad s \in \mathbf{S}^{\text {IP }}$

which can be seen as a special case of Eq. (2). Note that the temperature difference is only for the classification purpose and does not affect the heat duty calculation. 


\section{Phase Changes}

In general, a process stream might undergo phase changes and therefore a single stream could involve up to three regions: liquid, vapor, and two-phase (Hasan, Jayaraman, Karimi, \& Alfadala, 2010; Kamath et al., 2012; Ponce-Ortega, Jiménez-Gutiérrez, \& Grossmann, 2008). If a stream undergoes phase changes, we can no longer assume constant heat capacity. A more accurate yet tractable approach is to assume constant heat capacity only within each region (Kamath et al., 2012). In other words, the T-H diagram is represented by line segments (see Figure 5). The process stream with phase changes will then be divided into sub-streams to represent the regions it spans so that each sub-stream has constant heat capacity. Note that if we assume that a process stream with phase changes contains only a pure substance and the stream pressure is fixed, then the phase changes should take place at a constant temperature, and therefore the sub-stream corresponding to the twophase region will be an isothermal stream. In this case we introduce a dummy $1 \mathrm{~K}$ difference between $T^{B B L}$ and $T^{D E W}$.

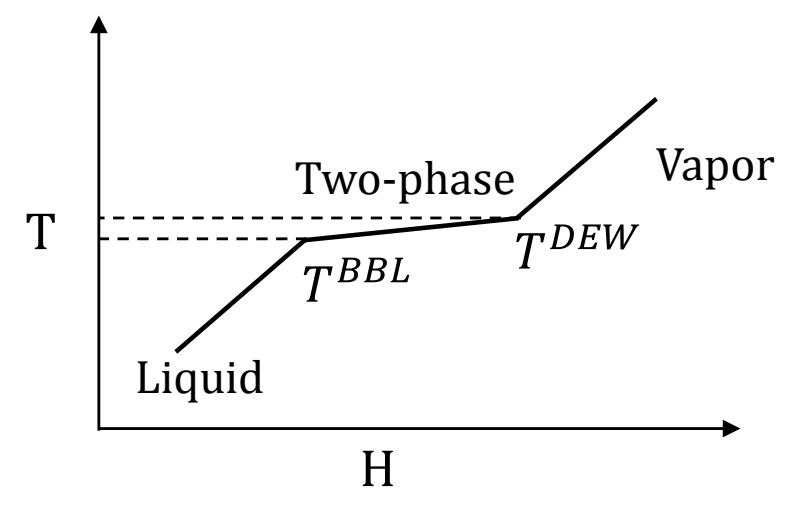

Figure 5. An example of piecewise linear representation of the T-H diagrams.

One of the major difficulties in modeling process streams with phase changes in the context of variable stream conditions is the detection of phase changes. Since the stream inlet and outlet temperatures are allowed to vary, the phases that a stream spans are unknown before solving the model. Moreover, the dew point and bubble point of a given stream are related to the stream composition and pressure, and they are therefore decision variables, which further increases the difficulty in determining what phases the stream spans. To address this issue, we propose a new disjunctive model for phase detection. Kamath et. al. (2012) proposed a disjunctive programming reformulation resulting into a nonlinear programming (NLP) model. Our reformulation results into mixed integer linear constraints, and it is formulated so that it can be integrated with the heat integration model proposed in this paper.

Given is a stream that potentially undergoes phase changes denoted as the "parent" stream; regardless of the phases it eventually spans, we first divide it into up to three sub-streams, LQ, VP, and $2 \mathrm{P}$, to represent the liquid, vapor, and two-phase regions, respectively. Since a given stream can start and end at one of the three regions, there are a total of nine possible cases (see Table 1 and Figure 6). 
Table 1. Nine possible cases for a process stream $s$ with potential phase changes. Subscript $s^{\prime}$ represents the sub-streams of process stream $s$.

\begin{tabular}{|c|c|c|c|c|c|c|c|c|c|c|}
\hline \multirow{2}{*}{ Case } & \multirow{2}{*}{ Start } & \multirow{2}{*}{ Finish } & \multirow{2}{*}{$\operatorname{TIN}_{s} \in$} & \multirow{2}{*}{$\operatorname{TOUT}_{s} \in$} & \multicolumn{3}{|c|}{$T_{T I N_{S^{\prime}}}$} & \multicolumn{3}{|c|}{ TOUT $_{s^{\prime}}$} \\
\hline & & & & & LQ & $2 \mathrm{P}$ & VP & LQ & $2 \mathrm{P}$ & VP \\
\hline 1 & LQ & LQ & {$\left[0, T^{B B L}\right]$} & {$\left[0, T^{B B L}\right]$} & TIN & 0 & 0 & TOUT & 0 & 0 \\
\hline 2 & LQ & $2 \mathrm{P}$ & {$\left[0, T^{B B L}\right]$} & {$\left[T^{B B L}, T^{D E W}\right]$} & TIN & $T^{B B L}$ & 0 & $T^{B B L}$ & TOUT & 0 \\
\hline 3 & LQ & VP & {$\left[0, T^{B B L}\right]$} & {$\left[T^{D E W}, \gamma^{U}\right]$} & TIN & $T^{B B L}$ & $T^{D E W}$ & $T^{B B L}$ & $T^{D E W}$ & TOUT \\
\hline 4 & $2 \mathrm{P}$ & LQ & {$\left[T^{B B L}, T^{D E W}\right]$} & {$\left[0, T^{B B L}\right]$} & $T^{B B L}$ & TIN & 0 & TOUT & $T^{B B L}$ & 0 \\
\hline 5 & $2 \mathrm{P}$ & $2 \mathrm{P}$ & {$\left[T^{B B L}, T^{D E W}\right]$} & {$\left[T^{B B L}, T^{D E W}\right]$} & 0 & TIN & 0 & 0 & TOUT & 0 \\
\hline 6 & $2 \mathrm{P}$ & VP & {$\left[T^{B B L}, T^{D E W}\right]$} & {$\left[T^{D E W}, \gamma^{U}\right]$} & 0 & TIN & $T^{D E W}$ & 0 & $T^{D E W}$ & TOUT \\
\hline 7 & VP & LQ & {$\left[T^{D E W}, \beta^{U}\right]$} & {$\left[0, T^{B B L}\right]$} & $T^{B B L}$ & $T^{D E W}$ & TIN & TOUT & $T^{B B L}$ & $T^{D E W}$ \\
\hline 8 & VP & $2 \mathrm{P}$ & {$\left[T^{D E W}, \beta^{U}\right]$} & {$\left[T^{B B L}, T^{D E W}\right]$} & 0 & $T^{D E W}$ & TIN & 0 & TOUT & $T^{D E W}$ \\
\hline 9 & VP & VP & {$\left[T^{D E W}, \beta^{U}\right]$} & {$\left[T^{D E W}, \gamma^{U}\right]$} & 0 & 0 & TIN & 0 & 0 & TOUT \\
\hline
\end{tabular}

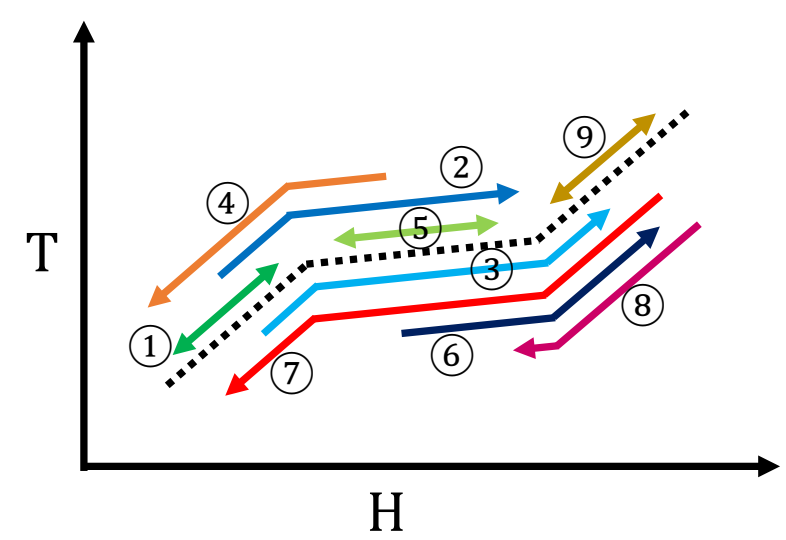

Figure 6. A graphic representation of the nine cases (numbered in circles) from Table 1. Each solid line represents one distinct case. Note that the lines are shifted vertically for better illustration.

Depending on the regions where the parent stream starts and ends (see the $2^{\text {nd }}$ and $3^{\text {rd }}$ columns in Table 1), which can be inferred by comparing the parent stream inlet/outlet temperatures with the dew point and bubble point (see the $4^{\text {th }}$ and $5^{\text {th }}$ columns), we enforce the sub-stream inlet and outlet temperatures as follows:

- If the parent stream starts/ends in a particular region, the inlet/outlet temperature of the sub-stream corresponding to this region will be equal to the parent stream inlet/outlet temperature, respectively;

- If the parent stream crosses the boundary of a region, the inlet and/or outlet temperature of the corresponding sub-stream will be set to $T^{B B L}$ or $T^{D E W}$; and

- If the parent stream does not exist at a region, both inlet and outlet temperatures of that substream will be set equal to zero.

To illustrate, we consider case 2 in which the stream starts as a liquid and ends in the two-phase region. In this case, the parent stream inlet temperature is no greater than the bubble point $\left(\operatorname{TIN}_{S} \leq\right.$ $T^{B B L}$ ) while the outlet temperature must be in between the bubble point and dew point $\left(T^{B B L} \leq\right.$ TOUT $_{S} \leq T^{D E W}$ ). We assign the parent stream inlet and outlet temperatures to the liquid sub-stream inlet $\left(\operatorname{TIN}_{S}=\operatorname{TIN}_{L Q}\right)$ and two-phase sub-stream outlet temperatures $\left(\operatorname{TOUT}_{S}=\operatorname{TOUT}_{2 P}\right)$, 
respectively. Further, temperatures of both the liquid sub-stream outlet and two-phase sub-stream inlet are set to the bubble point $\left(\operatorname{TIN}_{2 P}=\operatorname{TOUT}_{L Q}=T^{B B L}\right)$. Finally, we force inlet and outlet temperatures of the irrelevant vapor sub-stream to zero $\left(T I N_{V P}=T O U T_{V P}=0\right)$. This approach ensures that all the relevant sub-stream temperatures are correctly positioned with respect to the dew point and bubble point, and the heat loads used in the heat integration model are properly calculated using the approach discussed in Section 2.4.

The complete mixed-integer formulation of the logic conditions shown in Table 1 is given in Appendix A. Here, to illustrate the basic ideas, we show how to relate the parent stream inlet/outlet temperature to those of the sub-streams.

Given a subset of process streams that potentially undergo phase changes ( $s \in \mathbf{S}^{\mathbf{P C}}$ ), we first identify the regions that each stream can go through and define a set of sub-streams $\left(s^{\prime} \in \mathbf{S}_{s}^{\text {SUB }}\right)$ only for these regions. Then, we introduce binary variables $U_{s^{\prime}}$ and $V_{s^{\prime}}$, defined over $s^{\prime} \in \mathbf{S}_{s}^{\text {SUB }}$, to represent the starting and ending regions, respectively. Since a given parent stream $(s)$ can only start and end at one of its sub-streams, the following constraints are enforced:

$\sum_{s^{\prime} \in \mathbf{S}_{s}^{\text {SUB }}} U_{s^{\prime}}=1$

$s \in \mathbf{S}^{\mathbf{P C}}$

$\sum_{S^{\prime} \in \mathbf{S}_{s}^{\text {SUB }}} V_{s^{\prime}}=1$

$s \in \mathbf{S}^{\mathbf{P C}}$

Next we disaggregate the inlet and outlet temperatures of the parent streams into sub-streams (see Eqs. (36) and (37)). Note that $T I N_{s^{\prime}}^{P}$ and $T O U T_{s^{\prime}}^{P}$ are only used here to relate the parent stream and sub-stream inlet and outlet temperatures, and they are different from the sub-stream inlet and outlet temperatures from Table 1. Here Eqs. (38) and (39) ensure that $T I N_{s^{\prime}}^{P}$ and $T O U T_{s^{\prime}}^{P}$ are equal to zero when the corresponding binary variables are zero, and Eqs. (40) - (43) disaggregate the sub-stream inlet and outlet temperatures into hot/cold counter-parts and into temperature intervals if and only if the corresponding binaries are equal to 1 . Thus, the parent stream temperatures are only assigned to the correct sub-streams.

$$
\begin{array}{ll}
\operatorname{TIN}_{s}=\sum_{s^{\prime} \in \mathbf{S}_{s}^{\text {SUB }} \operatorname{TIN}_{s^{\prime}}^{P}} & s \in \mathbf{S}^{\mathbf{P C}} \\
\operatorname{TOUT}_{s}=\sum_{s^{\prime} \in \mathbf{S}_{s}^{\text {SUB }}} \operatorname{TOUT}_{s^{\prime}}^{P} & s \in \mathbf{S}^{\mathbf{P C}} \\
\operatorname{TIN}_{s^{\prime}}^{P} \leq \beta_{s^{\prime}}^{U} U_{s^{\prime}} & s \in \mathbf{S}^{\mathbf{P C}}, s^{\prime} \in \mathbf{S}_{s}^{\text {SUB }} \\
\operatorname{TOUT}_{s^{\prime}}^{P} \leq \gamma_{s^{\prime}}^{U} V_{s^{\prime}} & s \in \mathbf{S}^{\mathbf{P C}}, s^{\prime} \in \mathbf{S}_{s}^{\text {SUB }} \\
\operatorname{TIN}_{s^{\prime}}^{P} \leq \sum_{k}\left(\operatorname{TIN}_{s^{\prime}, k}^{C}+\operatorname{TIN}_{s^{\prime}, k}^{H}\right)+\beta_{s^{\prime}}^{U}\left(1-U_{s^{\prime}}\right) & s \in \mathbf{S}^{\mathbf{P C}}, s^{\prime} \in \mathbf{S}_{s}^{\text {SUB }} \\
\operatorname{TIN}_{s^{\prime}}^{P} \geq \sum_{k}\left(\operatorname{TIN}_{s^{\prime}, k}^{C}+\operatorname{TIN}_{s^{\prime}, k}^{H}\right)-\beta_{s^{\prime}}^{U}\left(1-U_{s^{\prime}}\right) & s \in \mathbf{S}^{\mathbf{P C}}, s^{\prime} \in \mathbf{S}_{s}^{\text {SUB }} \\
\operatorname{TOUT}_{s^{\prime}}^{P} \leq \sum_{k}\left(\operatorname{TOUT}_{s^{\prime}, k}^{C}+\operatorname{TOUT}_{s^{\prime}, k}^{H}\right)+\gamma_{s^{\prime}}^{U}\left(1-V_{s^{\prime}}\right) & s \in \mathbf{S}^{\mathbf{P C}}, s^{\prime} \in \mathbf{S}_{s}^{\text {SUB }} \\
\operatorname{TOUT}_{s^{\prime}}^{P} \geq \sum_{k}\left(\operatorname{TOUTT}_{s^{\prime}, k}^{C}+\operatorname{TOUT}_{s^{\prime}, k}^{H}\right)-\gamma_{s^{\prime}}^{U}\left(1-V_{s^{\prime}}\right) & s \in \mathbf{S}^{\mathbf{P C}}, s^{\prime} \in \mathbf{S}_{s}^{\text {SUB }}
\end{array}
$$

It is important to note that although sub-streams are originated from their parent streams, they are treated as independent streams in the heat integration model. When counting the required intervals, 
we should consider the number of sub-streams instead of the number of parent streams. Each substream has its own hot/cold identity, which is consistent with that of its parent stream, through temperature relationships. Therefore, the extension to phase change can also handle unclassified process streams and is compatible with any aforementioned extensions.

\section{Solution Methods}

The proposed methodology leads to an MINLP problem, which can be difficult to solve. In addition, the extensions discussed in the previous section further increase the size and complexity of the model. Accordingly, we develop solution methods, specifically, preprocessing to reduce the size of the model. The preprocessing steps are based on the stream information (e.g. hot/cold identity, and bounds on temperatures) that is available.

\subsection{Stream Hot/Cold Classification}

Since most of the related variables such as temperatures and heat duties have to be disaggregated, the resulting model is large. If some of the streams can be classified a priori as hot or cold, then we can fix some of the disaggregated variables e.g., if a stream is known to be a hot stream, $W_{s}^{H}$ is fixed to one and all the variables associated to that stream with a superscript " $\mathrm{C}$ " are fixed to zero.

Further, we can divide the set of streams into three subsets: (1) preclassified hot streams $\left(s \in \mathbf{S}^{\mathbf{H}}\right)$, (2) preclassified cold streams $\left(s \in \mathbf{S}^{\mathbf{C}}\right)$, and (3) unclassified streams $\left(s \in \mathbf{S}^{\mathbf{U N}}\right)$. While the set of unclassified streams are modeled using constraints in the proposed model, we remove and modify constraints for the preclassified streams:

- If a stream is preclassified as hot, we remove constraints with a "C" suffix, and we replace variable $W_{s}^{H}$ by 1 in Eqs. (3), (7H), and (13H), and

- If a stream is prelasssified as cold, we remove constraints with an " $\mathrm{H}$ " suffix, and we replace variable $W_{s}^{C}$ by 1 in Eqs. (4), (7C), and (13C)

\subsection{Stream Temperatures}

\subsubsection{Inlet Temperatures}

During preprocessing, we take advantage of the information on stream inlet temperatures. For example, some of the assignments might be readily available prior to solving the problem by analyzing the bounds on stream inlet temperatures. If stream $s$ is hot and $\operatorname{TIN}_{S}$ can be determined a priori as the $k^{\prime}$ th highest among all the streams, then we can set $X_{s, k^{\prime}}^{H}=1, T_{k^{\prime}}=\operatorname{TIN}_{s, k^{\prime}}^{H}=T I N_{s}$, and $X_{s, k}^{H}=T I N_{s, k}^{H}=0$ for all $k \neq k^{\prime}$. Also when the inlet temperature of a hot stream is $T_{k^{\prime}}$, the stream cannot end or span intervals above $k^{\prime}$, and thus we fix binary variables $\left(Y_{s, k}^{H}\right.$ and $\left.Z_{s, k}^{H}\right)$ and the corresponding continuous variable $\left(\operatorname{TOUT}_{s, k}^{H}, Q 1_{s, k}^{H}\right.$, and $Q 2_{s, k}^{H}$ ) to zero for all $\forall k \leq k^{\prime}$. Further, Eqs. $(18 \mathrm{H})$ and $(20 \mathrm{H})-(26 \mathrm{H})$ for stream $s$ are only written for $k>k^{\prime}$.

When the inlet temperature for stream $s$ is constant (i.e., $T I N_{s}=\Gamma_{S}$ ), we do not need to disaggregate the inlet temperature for stream $s$. In other words, we do not define $\operatorname{TIN}_{s, k}^{H}$ or $T I N_{s, k}^{C}$ for stream $s$. 
Further, we can reduce the number of constraints by replacing Eqs. (9) - (12C) with the following constraints for stream $s$ :

$T_{k} \geq \Gamma_{s}-\bar{T}_{k}\left(1-X_{s, k}^{H}\right) \quad k \in \mathbf{K}$

$T_{k} \leq \Gamma_{s}+\bar{T}_{k}\left(1-X_{s, k}^{H}\right) \quad k \in \mathbf{K}$

$T_{k} \geq \Gamma_{s}+\delta-\bar{T}_{k}\left(1-X_{s, k}^{C}\right) \quad k \in \mathbf{K}$

$T_{k} \leq \Gamma_{s}+\delta+\bar{T}_{k}\left(1-X_{s, k}^{C}\right) \quad k \in \mathbf{K}$

where $\bar{T}_{k}$ is a valid upper bound on $T_{k}$. The above constraints enforce that $T_{k}=\Gamma_{s}=$ TIN $N_{S}$ if $X_{s, k}^{H}=$ 1 , and $T_{k}-\delta=\Gamma_{S}=\operatorname{TIN}_{s}$ if $X_{s, k}^{C}=1$.

\subsubsection{Outlet Temperatures}

Similarly, we can compare bounds on stream outlet temperatures with the bounds on interval temperatures to obtain information regarding the location of the outlet temperatures in order to fix some of the $Y_{s, k}^{H} / Y_{s, k}^{C}$ variables and the related continuous variables (see Appendix B).

If the outlet temperature for stream $s$ is constant $\left(\Theta_{s}\right)$, then we do not need to disaggregate the outlet temperature or define $\operatorname{TOUT}_{s, k}^{H} / \operatorname{TOUT}_{S, k}^{C}$. We can replace Eqs. (14) - (17C) by the following equations:

$T_{k-1} \geq \Theta_{s}-\bar{T}_{k}\left(1-Y_{s, k}^{H}\right) \quad k \in \mathbf{K}$

$T_{k} \leq \Theta_{s}+\bar{T}_{k}\left(1-Y_{s, k}^{H}\right) \quad k \in \mathbf{K}$

$T_{k-1} \geq \Theta_{s}+\delta-\bar{T}_{k}\left(1-Y_{s, k}^{C}\right) \quad k \in \mathbf{K}$

$T_{k} \leq \Theta_{s}+\delta+\bar{T}_{k}\left(1-Y_{s, k}^{C}\right) \quad k \in \mathbf{K}$

which ensure that $\operatorname{TOUT}_{s}=\Theta_{s} \in\left[T_{k}, T_{k-1}\right]$ if $Y_{s, k}^{H}=1$ and $T O U T_{s}=\Theta_{s} \in\left[T_{k}-\delta, T_{k-1}-\delta\right]$ if $Y_{s, k}^{C}=$ 1. Note that since $\operatorname{TOUT}_{S, k}^{H} / \operatorname{TOUT}_{s, k}^{C}$ are not defined here, we also need to replace $T O U T_{s, k}^{H}$ and TOUT $_{s, k}^{C}$ in Eqs. $(24 \mathrm{H})$ and (24C), respectively, with TOUT $T_{s}$ for stream $s$.

Finally, if all the flow rates or all the stream temperatures are constants, the proposed model is reduced to a MILP problem. In the extreme case that all streams have known hot/cold classification, and both temperatures and flow rates are constants, the model becomes the LP transshipment model. Detailed preprocessing algorithms for the cases discussed here are shown in Appendix B.

\section{Integration with Process Synthesis}

While the unit operations can be modeled using various methods (Bausa, Von Watzdorf, \& Marquardt, 1998; Boukouvala \& Ierapetritou, 2013; Gani \& Bek - Pedersen, 2000; Kong, Sen, Henao, Dumesic, \& Maravelias, 2016), in this work we adopt a "flow based" formulation (Kong et al., 2016; Wu, Henao, \& Maravelias, 2016). First, a unit selection binary variable $\left(Y_{i}^{U N I T}\right)$ is introduced for each alternative unit $(i \in \mathbf{I})$ so that $Y_{i}^{U N I T}=1$ if the unit is selected. If the unit is not selected $\left(Y_{i}^{U N I T}=0\right)$, we force the inlet component flows of that unit to zero. Note that according to the approach of Kong et al. 
(2016), if the unit inlet component flows are equal to zero, not only the unit outlet flows are forced to zero by material balances, the heat duty and cost of that unit are also forced to zero.

In the process model, we define a set of streams $(s \in \mathbb{S})$ that connect the inlet and outlet between two processing units or supply/remove materials to/from the process. The set of process streams used in the heat integration model $\left(\mathbf{S}^{\mathbf{P}}\right)$ consists of a subset of $\mathbb{S}$ which potentially requires heating/cooling, and a set of streams representing the heat duties of processing units. The process model is linked to the heat integration model via linking variables: $T I N_{S}, T O U T_{s}$, and $F_{S}$ for all $s \in \mathbf{S}^{\mathbf{P}}$ (see Figure 7). They are simultaneously determined and optimized by the process and the heat integration. Stream temperatures, $T_{I} N_{S}$ and $T O U T_{S}$, are related to the temperatures of the units that it connects (see Figure 8). If a subset of streams potentially undergo phase changes, the extension to phase changes ensures that the inlet and outlet temperatures of each sub-streams are correctly calculated when entering the heat integration model.

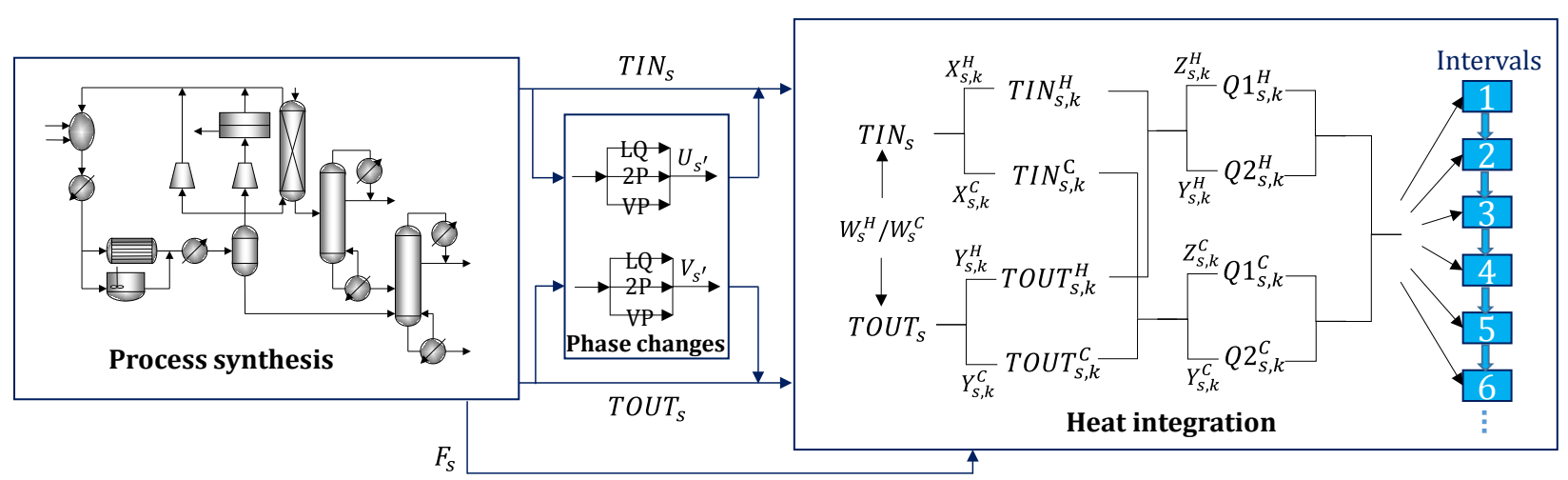

Figure 7. Integrating process synthesis and heat integration.

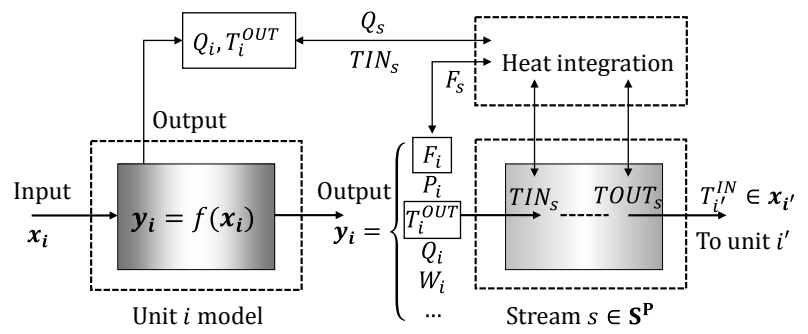

Figure 8. Relationship between stream temperatures and unit models. Vectors $\boldsymbol{x}_{\boldsymbol{i}}$ and $\boldsymbol{y}_{\boldsymbol{i}}$ are all the input and output variables to unit model $i$, respectively.

Finally, to reduce the cardinality of $\mathbf{S}^{\mathbf{P}}$ and thus the number of temperature intervals, which will in turn lead to smaller models, we carefully define the subset of process streams. The idea is illustrated in Figure 9, where the original superstructure representation with $\mathbf{S}^{\mathbf{P}}=\{2,3,4,5\}$ is replaced with an alternative representation with $\mathbf{S}^{\mathbf{P}}=\{1,5\}$. Note that the two representation have the same feasible solutions embedded on them. 

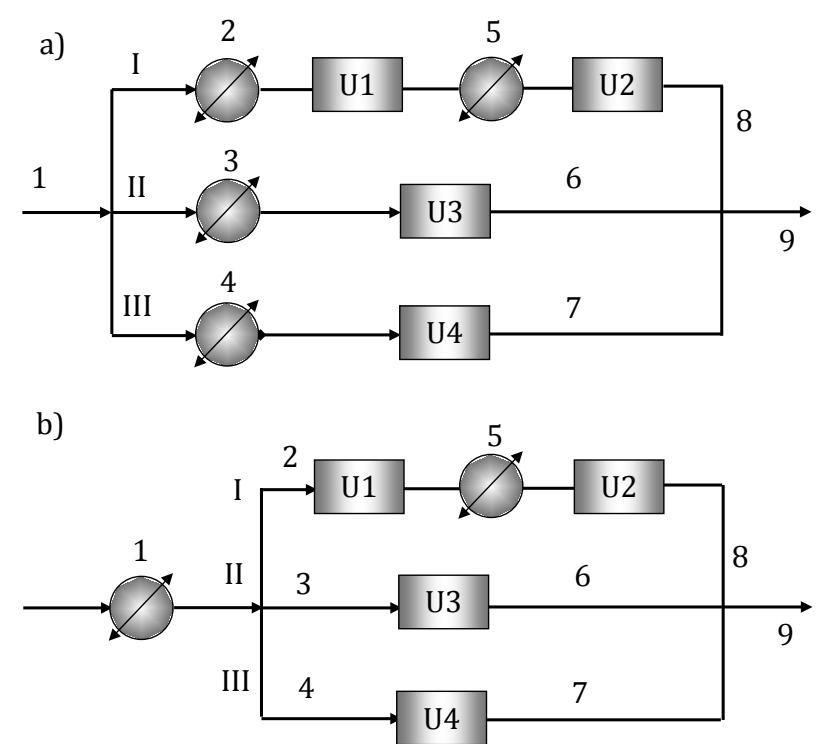

Figure 9. Superstructure example with four units (U1 - U4) and nine streams (1 - 9). Only one of the three alternatives (I, II, and III) can be selected in any feasible solution. Representation (a) includes four streams that require heating/cooling, while (b) includes only two process streams with heating/cooling.

\section{Examples}

The applicability of the proposed model is first illustrated through two illustrative examples. The first example includes a process stream with potential phase changes, while the second considers multiple utilities and isothermal process streams. Then in Section 7.2, we consider an example with simultaneous process synthesis and heat integration. In all three examples, we assume that a hot utility is available at $500{ }^{\circ} \mathrm{C}$ with a unit cost of $\$ 80 / \mathrm{kW}$-yr and a cold utility enters at $20^{\circ} \mathrm{C}$ and exits at $30^{\circ} \mathrm{C}$ with a cost of $\$ 20 / \mathrm{kW}$-yr. In the two small examples, the only nonlinearities are the product of flow rate and temperature difference in the heat duty calculation (Eqs. $(22 \mathrm{H}),(22 \mathrm{C}),(24 \mathrm{H})$, and $(24 \mathrm{C})$ ) and the product of flow rate and latent heat (Eq. (32)) if isothermal streams are involved. In the last example, the process model leads to additional nonlinearities including bilinear terms in the energy balance, exponential functions in the reaction calculations, and power functions in the cost estimations. All three examples are formulated in GAMS (24.7.1) and solved using BARON (16.3.4) (Tawarmalani \& Sahinidis, 2005) on a desktop with $3.60 \mathrm{GHz}$ Intel i7 processors.

\subsection{Illustrative Examples}

\subsubsection{Example 1}

The first example has five process streams and two utilities. One of the process streams (Stream 5) has the potential to undergo phase changes, so it is divided into three sub-streams (5-LQ, 5-2P, and 5 -VP) and the parent stream is disregarded. The bubble point and dew point of this stream is assumed to be $320^{\circ} \mathrm{C}$ and $335^{\circ} \mathrm{C}$, respectively. We assume constant $T_{S}^{B B L}$ and $T_{S}^{D E W}$ in this example so that the constraints in appendix A.2 remain linear. To address problems where $T_{S}^{B B L}$ and $T_{s}^{D E W}$ are unknown, they are defined as variables, and a reformulation is required to retain linearity. Due 
to the differences in heat capacities, the heat capacity flows of the sub-streams in this example follow: $2 F_{5-L Q}=F_{5-2 P}=4 F_{5-V P}$. The final model has 7 process streams, whose specifications are shown in Table 2.

Table 2. Stream specifications for Example 1

\begin{tabular}{ccccc}
\hline Stream \# & Class & $\begin{array}{c}\text { TIN range } \\
\left({ }^{\circ} \mathrm{C}\right)\end{array}$ & $\begin{array}{c}\text { TOUT range } \\
\left({ }^{\circ} \mathrm{C}\right)\end{array}$ & $\begin{array}{c}\text { Flow range } \\
\left(\mathrm{MW} /{ }^{\circ} \mathrm{C}\right)\end{array}$ \\
\hline 1 & Hot & $400-440$ & $110-130$ & 1 \\
2 & Hot & $340-380$ & $120-130$ & $0.8-1.1$ \\
3 & Cold & $160-180$ & $400-410$ & 0.7 \\
4 & Unknown & $240-270$ & $250-260$ & 0.9 \\
$5-L Q$ & Unknown & $307-405$ & $331-337$ & $1.8-2$ \\
5-2P & Unknown & $307-405$ & $331-337$ & $3.6-4$ \\
5 5P & Unknown & $307-405$ & $331-337$ & $0.9-1$ \\
\hline
\end{tabular}

The first example has eight temperature intervals and a heat recovery approach temperature of $20^{\circ} \mathrm{C}$. The problem contains 2120 constraints and 1462 variables, including 381 binary variables. The model is solved to optimality in 165 seconds. The optimal stream temperatures and flow rates are shown in Figure 10 and Table 3.

Table 3. Optimal stream conditions for Example 1

\begin{tabular}{ccccc}
\hline Stream \# & Class & $\begin{array}{c}\text { TIN } \\
\left({ }^{\circ} \mathrm{C}\right)\end{array}$ & $\begin{array}{c}\text { TOUT } \\
\left({ }^{\circ} \mathrm{C}\right)\end{array}$ & $\begin{array}{c}\text { Flow } \\
\left(\mathrm{MW} /{ }^{\circ} \mathrm{C}\right)\end{array}$ \\
\hline 1 & Hot & 420 & 130 & 1 \\
2 & Hot & 340 & 130 & 0.8 \\
3 & Cold & 160 & 400 & 0.7 \\
4 & Cold & 240 & 260 & 0.9 \\
$5-\mathrm{LQ}$ & - & - & - & - \\
5-2P & Cold & 331 & 335 & 4 \\
$5-\mathrm{VP}$ & Cold & 335 & 337 & 1 \\
\hline
\end{tabular}

In the optimal solution, both of the unclassified streams (stream 4 and 5) are cold streams. Stream 5 enters at the two-phase region at $331^{\circ} \mathrm{C}$ and exits as a vapor at $337^{\circ} \mathrm{C}$. Since it does not span the liquid phase, both inlet and outlet temperatures of sub-stream 5-LQ are forced to zero. The process requires $254 \mathrm{MW}$ of cooling while no external heating is required. The total utility cost is $\$ 50.8 \mathrm{MM} / \mathrm{yr}$. 


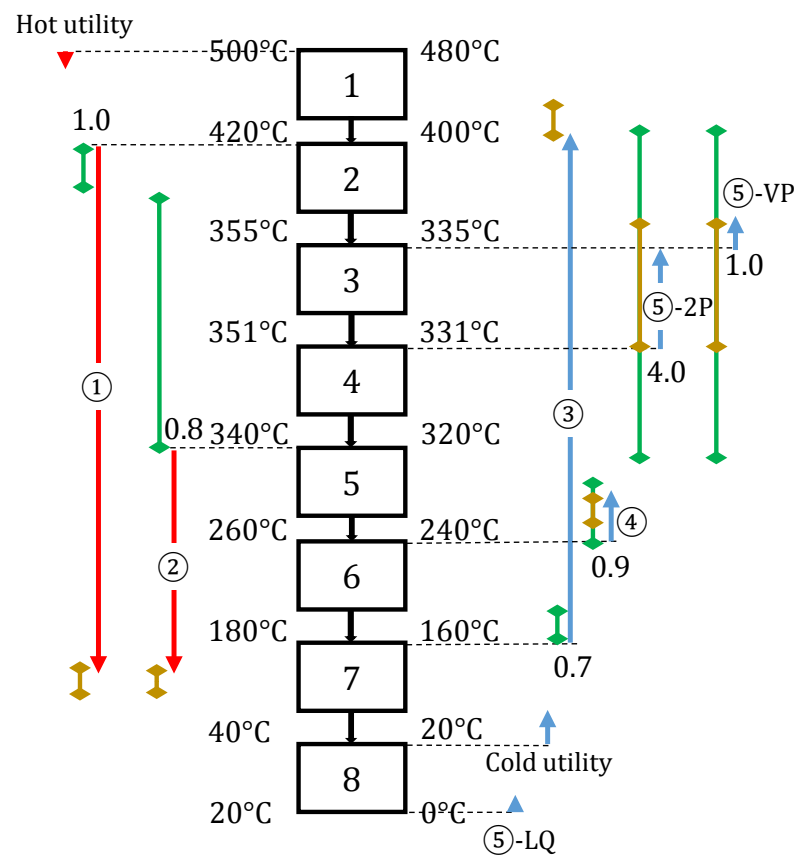

Figure 10. Stream temperatures of optimal solution of Example 1. Red and blue arrows represent hot and cold streams, respectively. The green and orange lines on their immediate left represent the bounds on the stream inlet and outlet temperatures, respectively. Optimum stream flow rates are shown at the tail of the arrows.

\subsubsection{Example 2}

The second example includes 17 process streams: 6 hot streams, 7 cold streams, and 4 unclassified streams. The bounds on temperatures and flow rates are shown in Table 4.

Stream \#2 is an isothermal stream while all other streams are non-isothermal. In addition to the hot utility at $500{ }^{\circ} \mathrm{C}$, here we assume that a second hot utility is available at $380{ }^{\circ} \mathrm{C}$ with a unit cost of $\$ 60 / \mathrm{kW}$-yr. Nineteen temperature intervals are defined and a heat recovery approach temperature of $20^{\circ} \mathrm{C}$ is assumed. The model includes 9714 constraints and 5801 variables, of which 2083 are binary variables. The problem is solved to optimality in 13275 seconds. The optimal temperatures and flow rates are shown in Table 4. 
Table 4. Stream specifications and optimal solutions for Example 2

\begin{tabular}{ccccc}
\hline Stream \# & Class & TIN $\left({ }^{\circ} \mathrm{C}\right)$ & TOUT $\left({ }^{\circ} \mathrm{C}\right)$ & $\begin{array}{c}\text { Flow } \\
\left(\mathrm{MW} /{ }^{\circ} \mathrm{C}\right)\end{array}$ \\
\hline 1 & Hot & $440(400-440)$ & $130(110-130)$ & 1 \\
2 & Hot & $340(340-380)$ & $340(340-380)$ & $100 \mathrm{MW}$ \\
3 & Cold & $180(160-180)$ & $415(415-425)$ & $3(3-4)$ \\
4 & Cold & $110(100-120)$ & $250(250-260)$ & $3(3-4)$ \\
5 & Hot & $240(130-240)$ & $190(150-300)$ & 1 \\
6 & Cold & $430(180-430)$ & $210(210-300)$ & 2 \\
7 & Hot & 280 & 140 & $2(1.5-2)$ \\
8 & Hot & 355 & $190(190-200)$ & $1.3(1.1-1.3)$ \\
9 & Cold & $410(360-410)$ & 411 & $3.3(3.3-4)$ \\
10 & Cold & 230 & 320 & $3(3-3.5)$ \\
11 & Cold & 390 & 460 & 0.9 \\
12 & Cold & $160(150-160)$ & $170(120-180)$ & 3 \\
13 & Hot & 220 & $180(170-180)$ & $1(0.5-1)$ \\
14 & Cold & 300 & $400(400-408)$ & 1.6 \\
15 & Cold & 170 & $440(440-450)$ & 3.5 \\
16 & Hot & 480 & $440(440-460)$ & 1.8 \\
17 & Hot & $190(170-190)$ & 180 & $4(3.2-4)$ \\
\hline
\end{tabular}

Bounds on temperatures and flow rates are shown in parentheses following the optimal solutions. Streams $\# 5,6,12$, and 17 are unclassified a priori. Stream \#2 is assumed isothermal.

After heat integration, the $1049.8 \mathrm{MW}$ of heating is satisfied by hot utility (190.3 MW) and intermediate hot utility (859.5 MW), while no cooling is required. The total utility cost is found to be $\$ 66.8 \mathrm{MM} / \mathrm{yr}$.

\subsection{Simultaneous Process Synthesis and Heat Integration}

\subsubsection{Problem Statement and Assumptions}

Four main components (A, B, C, and D) are involved in the process. Components A and B are used as raw materials to produce component $C$ in the first reaction (RXN 1), which can be carried out in two alternative isothermal continuous stir-tank reactors (CSTR1 and CSTR2) employing different catalysts. The effluent stream of the reactor is sent to a flash tank to separate unreacted A and B from intermediate C. Unless stated otherwise, the separations in this example are assumed sharp (i.e., all the light components exit at the top while all the heavy components come out from the bottom). Unreacted A and B are collected at the top and recycled, while the bottom of the flash contains pure C, which is sent to another isothermal continuous stir-tank reactor (CSTR3) to produce final product D. This second reaction (RXN 2) is assumed to be an equilibrium reaction, and the equilibrium constant is a function of temperature (see Appendix C for details). The reaction is assumed endothermic and therefore the reactor requires heating. Finally, unreacted C is separated from D in one of three alternative separation technologies before recycled back to CSTR3. Table 5 summarizes the critical parameters for each unit in the superstructure. Each unit has a cost which is approximated by a power-law function of the total inlet flow. It is assumed that the feed stream (stream 1) flow rates are $2 \mathrm{kmol} / \mathrm{s}$ of $A$ and $1 \mathrm{kmol} / \mathrm{s}$ of $B$, and we are selling final product $D$ at a price 
of $\$ 1700 /$ ton. The objective is to maximize profit, which takes into account the revenue, cost of materials, unit capital cost, and utility cost.

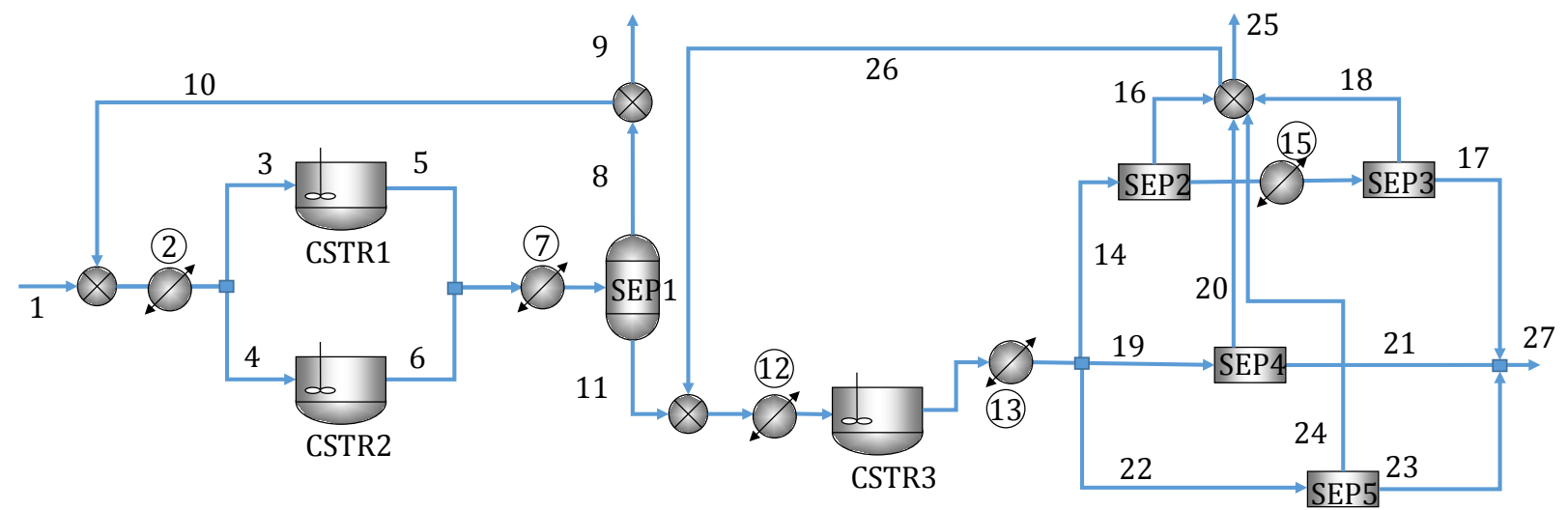

Figure 11. A superstructure chemical process. Streams in the chemical process $(s \in \mathbb{S})$ are numbered. Streams numbered in circles are process streams that potentially require heat/cooling.

Table 5. Unit specifications for superstructure example

\begin{tabular}{ccccc}
\hline Reactors & RXN & $\begin{array}{c}\text { Temperature } \\
(\mathrm{K})\end{array}$ & Conversion & $\begin{array}{c}\text { Unit cost } \\
\text { pre-factor, } \kappa \\
\left(\$ \text { mol-0.6yr }^{-0.4}\right)\end{array}$ \\
\hline CSTR 1 & $A+B \rightarrow C$ & 500 & 0.9 & 0.9 \\
CSTR 2 & $A+B \rightarrow C$ & 400 & 0.8 & 0.85 \\
CSTR 3 & $C \rightleftharpoons D$ & $330-400$ & variable & 1 \\
\hline Separators & Top/Bottom & & - & 1 \\
\hline SEP 1 & AB/C & 430 & & 1.1 \\
SEP 2 & C/D & 380 & & 1.1 \\
SEP 3 & C/D & 340 & - & 0.9 \\
SEP 4 & C/D & 360 & & 0.8 \\
SEP 5 & C/D & 350 & & .
\end{tabular}

The split fractions in SEP 2 are 0.6 and 0 for component $C$ and D, respectively. The conversion is with respect to the limiting component B. Cost pre-factor $\kappa$ relates the total molar flow at the inlet $\left(F_{i}^{T}\right)$ to the annualized cost: $C_{i}=\kappa_{i}\left(F_{i}^{T}\right)^{0.6}$.

There are a total of five process streams that require heating or cooling $(\{2,7,12,13,15\}$ in Figure 12$)$. Together with two isothermal streams that represent the heat duties of SEP1 and CSTR3, the total number of streams in the heat integration is seven, leading to eight temperature intervals. Due to the selection of alternative units and recycling, the hot/cold identities of streams 2, 7, 12, and 13 are unknown a priori.

\subsubsection{Results}

The resulting MINLP model consists of 1267 variables (298 binaries) and 1645 constraints. It was solved to global optimality in 34.2 seconds with an objective of $\$ 14.5 \mathrm{MM} / \mathrm{yr}$. CSTR1 is selected for the first reaction, and RXN1 takes place at $500 \mathrm{~K}$ with a 0.9 conversion of reactant $\mathrm{B}$. The effluent stream is subsequently sent to a flash tank operating at $430 \mathrm{~K}$. After mixing with the recycle stream, 
intermediate $\mathrm{C}$ is converted to D in CSTR3 at $386 \mathrm{~K}$. The equilibrium constant is determined to be 0.84 and the single-pass conversion of $\mathrm{C}$ in RXN2 is 0.46 . Following the second reaction, SEP5 is selected for the final product purification. The final product D is produced at a rate of $0.92 \mathrm{kmol} / \mathrm{s}$, leading to an overall yield of $92 \%$ with respect to limiting reactant B. Optimal stream conditions are shown in Tables C1 and C2 in the Appendix. After heat integration, the process requires $14 \mathrm{MW}$ external heating and while no cooling is required.

Note that while the recycle ratio for the first reaction is 0.18 , all the unreacted component $\mathrm{C}$ are recycled back to CSTR3 to maximize production of D. Another interesting observation is that after mixing with the recycle stream, the feed to CSTR3 is already at the reactor operating temperature, which makes stream 12 redundant in the heat integration (see Figure 12). This shows that our model is not only able to minimize energy consumption through heat integration between process streams, but also account for temperatures and energy flows of the overall process.

For comparison we generate a superstructure using the alternative representation shown in Figure 9a. Now the process streams that require heating/cooling are introduced right before the processing units and therefore the total number of process streams increases to 10 , which leads to 11 temperature intervals. The resulting model yields the same optimal cost, as expected, but it requires a solution time of 5231 seconds, which is one order-of-magnitude longer compared to that in the previous representation.

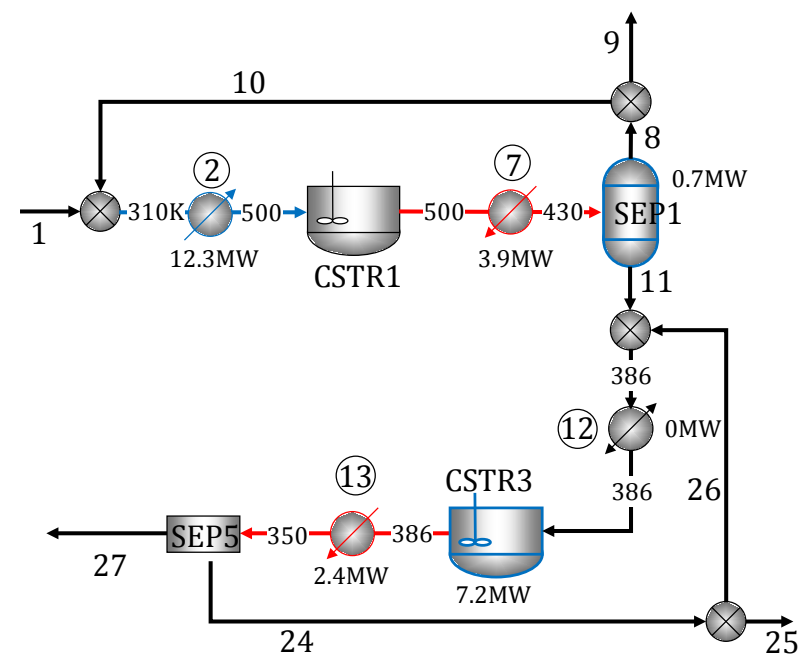

Figure 12. Process structure in the optimal solution. Hot/cold streams are shown in red/blue, respectively. The inlet and outlet temperatures (in Kelvin), and the heat duties are shown for all the streams that enter heat integration.

\section{Conclusions}

We proposed a targeting model that can be integrated with models for process synthesis. Importantly, the proposed model accounts for process streams that cannot be classified as hot or cold, a feature that often arises in process synthesis problems, but has not been addressed in the literature. The model is based on the introduction of stream classification binary variables and employs a "dynamic" 
temperature grid onto which (variable) stream inlet and outlet temperatures are mapped. Through temperature and heat duty variable disaggregation, we calculate heat duties for each stream in each interval. The model was extended to handle multiple utilities, isothermal and non-isothermal streams, and process streams with phase changes. Finally, a superstructure representation technique and several preprocessing methods were discussed to aid the solution process. The proposed methods allow us to address general process synthesis problems where the type of temperaturechanging operations are unknown prior to optimization.

\section{Acknowledgements}

This work was funded by the DOE Great Lakes Bioenergy Research Center (DOE BER Office of Science DE-FC02-07ER64494). 


\section{Appendix A. Streams with Phase Changes}

\section{A.1. An Alternative Formulation}

Here we present an alternative formulation to Eqs. (34) - (41). The parent stream inlet and outlet temperatures are disaggregated into intervals and sub-streams via Eqs. (A1) and (A2), respectively. Then, non-negative slack variables $\operatorname{TIN}_{s^{\prime}, k}^{D} / \operatorname{TOUT}_{s^{\prime}, k}^{D}$ and $T I N_{s^{\prime}, k}^{P} / T_{O U T} T_{s^{\prime}, k}^{P}$ are introduced, which are equal to zero if the corresponding binaries are equal to one:

$$
\begin{array}{lc}
\operatorname{TIN}_{s}=\sum_{k} \sum_{s^{\prime} \in \mathbf{S}_{S}^{\text {SUB }}} \operatorname{TIN}_{s^{\prime}, k}^{P} & s \in \mathbf{S}^{\mathbf{P C}} \\
\operatorname{TOUT}_{s}=\sum_{k} \sum_{s^{\prime} \in \mathbf{S}_{s}^{\text {SUB }} \operatorname{TOUT}_{s^{\prime}, k}^{P}} & s \in \mathbf{S}^{\mathbf{P C}} \\
\operatorname{TIN}_{s^{\prime}, k}^{P}+\operatorname{TIN}_{s^{\prime}, k}^{D}=\operatorname{TIN}_{s^{\prime}, k}^{C}+\operatorname{TIN}_{s^{\prime}, k}^{H} & s \in \mathbf{S}^{\mathbf{P C}}, s^{\prime} \in \mathbf{S}_{s}^{\mathbf{S U B}}, k \in \mathbf{K} \\
\operatorname{TIN}_{s^{\prime}, k}^{P} \leq \beta_{s^{\prime}}^{U} U_{s^{\prime}} & s \in \mathbf{S}^{\mathbf{P C}}, s^{\prime} \in \mathbf{S}_{s}^{\mathbf{S U B}}, k \in \mathbf{K} \\
\operatorname{TIN}_{s^{\prime}, k}^{D} \leq \beta_{s^{\prime}}^{U}\left(1-U_{s^{\prime}}\right) & s \in \mathbf{S}^{\mathbf{P C}}, s^{\prime} \in \mathbf{S}_{S}^{\mathbf{S U B}}, k \in \mathbf{K} \\
\operatorname{TOUT}_{s^{\prime}, k}^{P}+\operatorname{TOUT}_{s^{\prime}, k}^{D}=\operatorname{TOUT}_{s^{\prime}, k}^{C}+\operatorname{TOUT}_{s^{\prime}, k}^{H} & s \in \mathbf{S}^{\mathbf{P C}}, s^{\prime} \in \mathbf{S}_{S}^{\mathbf{S U B}}, k \in \mathbf{K} \\
\operatorname{TOUT}_{s^{\prime}, k}^{P} \leq \gamma_{s^{\prime}}^{U} V_{s^{\prime}} & s \in \mathbf{S}^{\mathbf{P C}}, s^{\prime} \in \mathbf{S}_{S}^{\mathbf{S U B}}, k \in \mathbf{K} \\
\operatorname{TOUT}_{s^{\prime}, k}^{D} \leq \gamma_{s^{\prime}}^{U}\left(1-V_{s^{\prime}}\right) & s \in \mathbf{S}^{\mathbf{P C}}, s^{\prime} \in \mathbf{S}_{s}^{\mathbf{S U B}}, k \in \mathbf{K}
\end{array}
$$

\section{A.2. Sub-stream Temperature Calculations}

In this section we compare the sub-stream inlet and outlet temperatures with the dew point and bubble point. All the constraints in this section, together with Eqs. (34) - (43), complete the formulation of the logic conditions as shown in Table 1 . We define binary variables $U_{s}^{L Q}, V_{s}^{L Q}, U_{S}^{2 P}$, $V_{s}^{2 P}$, and $U_{s}^{V P}, V_{s}^{V P}$, which are equivalent to $U_{s^{\prime}}$ and $V_{s^{\prime}}$ with $s^{\prime}$ representing the sub-streams of $s$; e.g., $U_{s}^{L Q} \equiv U_{s^{\prime} \in \mathbf{S}_{s}^{\mathbf{L Q}}}$

\section{Liquid phase}

$$
\begin{array}{ll}
\operatorname{TIN}_{s^{\prime}, k}^{H} \leq T_{s}^{B B L} V_{s}^{L Q} & s \in \mathbf{S}^{\mathbf{P C}}, s^{\prime} \in \mathbf{S}_{\boldsymbol{s}}^{\mathbf{L Q}}, k \in \mathbf{K} \\
\operatorname{TIN}_{s^{\prime}, k}^{C} \leq T_{s}^{B B L} U_{s}^{L Q} & s \in \mathbf{S}^{\mathbf{P C}}, s^{\prime} \in \mathbf{S}_{\boldsymbol{s}}^{\mathbf{L Q}}, k \in \mathbf{K} \\
\operatorname{TOUT}_{s^{\prime}, k}^{H} \leq T_{s}^{B B L} V_{s}^{L Q} & s \in \mathbf{S}^{\mathbf{P C}}, s^{\prime} \in \mathbf{S}_{\boldsymbol{s}}^{\mathbf{L Q}}, k \in \mathbf{K}^{\mathbf{I}} \\
\operatorname{TOUT}_{s^{\prime}, k}^{C} \leq T_{s}^{B B L}\left(U_{s}^{L Q}+V_{s}^{L Q}\right) & s \in \mathbf{S}^{\mathbf{P C}}, s^{\prime} \in \mathbf{S}_{\boldsymbol{s}}^{\mathbf{L Q}}, k \in \mathbf{K}^{\mathbf{I}} \\
\sum_{k} \operatorname{TIN}_{s^{\prime}, k}^{H} \leq T_{s}^{B B L}\left(2-V_{s}^{L Q}+U_{s}^{L Q}\right) & s \in \mathbf{S}^{\mathbf{P C}}, s^{\prime} \in \mathbf{S}_{\boldsymbol{s}}^{\mathbf{L Q}} \\
\sum_{k} \operatorname{TIN}_{s^{\prime}, k}^{H} \geq T_{s}^{B B L}\left(V_{s}^{L Q}-U_{s}^{L Q}\right) & s \in \mathbf{S}^{\mathbf{P C}}, s^{\prime} \in \mathbf{S}_{\boldsymbol{s}}^{\mathbf{L Q}} \\
\sum_{k} \operatorname{TOUT}_{s^{\prime}, k}^{C} \leq T_{s}^{B B L}\left(2-U_{s}^{L Q}+V_{s}^{L Q}\right) & s \in \mathbf{S}^{\mathbf{P C}}, s^{\prime} \in \mathbf{S}_{\boldsymbol{s}}^{\mathbf{L Q}} \\
\sum_{k} \operatorname{TOUT}_{s^{\prime}, k}^{C} \geq T_{s}^{B B L}\left(U_{s}^{L Q}-V_{s}^{L Q}\right) & s \in \mathbf{S}^{\mathbf{P C}}, s^{\prime} \in \mathbf{S}_{\boldsymbol{s}}^{\mathbf{L Q}}
\end{array}
$$

$\underline{\text { Two-phase region }}$

$$
\operatorname{TIN}_{s^{\prime}, k}^{H} \leq T_{s}^{D E W}\left(2-U_{s}^{L Q}-V_{s}^{L Q}\right) \quad s \in \mathbf{S}^{\mathbf{P C}}, s^{\prime} \in \mathbf{S}_{s}^{2 \mathbf{P}}, k \in \mathbf{K}
$$


$\operatorname{TIN}_{s^{\prime}, k}^{C} \leq T_{s}^{D E W}\left(2-U_{s}^{L Q}-V_{s}^{L Q}\right)$

$\operatorname{TOUT}_{s^{\prime}, k}^{H} \leq T_{s}^{D E W}\left(2-U_{s}^{L Q}-V_{s}^{L Q}\right)$

$\operatorname{TOUT}_{s^{\prime}, k}^{C} \leq T_{s}^{D E W}\left(2-U_{s}^{L Q}-V_{s}^{L Q}\right)$

$\operatorname{TIN}_{S^{\prime}, k}^{H} \leq T_{S}^{D E W}\left(2-U_{S}^{V P}-V_{S}^{V P}\right)$

$\operatorname{TIN}_{s^{\prime}, k}^{C} \leq T_{S}^{D E W}\left(2-U_{S}^{V P}-V_{S}^{V P}\right)$

$\operatorname{TOUT}_{s^{\prime}, k}^{H} \leq T_{s}^{D E W}\left(2-U_{s}^{V P}-V_{s}^{V P}\right)$

$\operatorname{TOUT}_{s^{\prime}, k}^{C} \leq T_{S}^{D E W}\left(2-U_{S}^{V P}-V_{S}^{V P}\right)$

$\sum_{k} T I N_{s^{\prime}, k}^{H} \leq T_{s}^{D E W}\left(2-U_{s}^{V P}+V_{s}^{V P}\right)$

$\sum_{k} T I N_{S^{\prime}, k}^{H} \geq T_{s}^{D E W}\left(U_{S}^{V P}-V_{S}^{V P}\right)$

$\sum_{k} \operatorname{TOUT}_{s^{\prime}, k}^{C} \leq T_{s}^{D E W}\left(2-V_{s}^{V P}+U_{s}^{V P}\right)$

$\sum_{k} \operatorname{TOUT}_{s^{\prime}, k}^{C} \geq T_{s}^{D E W}\left(V_{s}^{V P}-U_{s}^{V P}\right)$

$\sum_{k} \operatorname{TOUT}_{s^{\prime}, k}^{H} \leq T_{s}^{B B L}+T_{s}^{D E W}\left(1-V_{s}^{L Q}+U_{s}^{L Q}\right)$

$\sum_{k} \operatorname{TOUT}_{s^{\prime}, k}^{H} \geq T_{s}^{B B L}-T_{s}^{D E W}\left(1-V_{s}^{L Q}+U_{s}^{L Q}\right)$

$\sum_{k} \operatorname{TIN}_{s^{\prime}, k}^{C} \leq T_{s}^{B B L}+T_{s}^{D E W}\left(1-U_{s}^{L Q}+V_{s}^{L Q}\right)$

$\sum_{k} \operatorname{TIN}_{s^{\prime}, k}^{C} \geq T_{s}^{B B L}-T_{s}^{D E W}\left(1-U_{s}^{L Q}+V_{s}^{L Q}\right)$

$\sum_{k}\left(\right.$ TOUT $_{s^{\prime}, k}^{H}+$ TIN $\left._{s^{\prime}, k}^{C}\right) \geq T_{s}^{B B L}\left(1-U_{s}^{L Q}-V_{s}^{V P}\right)$

$\sum_{k}\left(\right.$ TOUT $_{s^{\prime}, k}^{H}+$ TIN $\left._{s^{\prime}, k}^{C}\right) \geq T_{s}^{B B L}\left(1-V_{s}^{L Q}-U_{s}^{V P}\right)$

$\sum_{k}\left(\right.$ TOUT $\left._{s^{\prime}, k}^{C}+\operatorname{TIN}_{s^{\prime}, k}^{H}\right) \geq T_{s}^{B B L}\left(1-U_{s}^{L Q}-V_{s}^{V P}\right)$

$\sum_{k}\left(\right.$ TOUT $\left._{s^{\prime}, k}^{C}+\operatorname{TIN}_{s^{\prime}, k}^{H}\right) \geq T_{s}^{B B L}\left(1-V_{s}^{L Q}-U_{S}^{V P}\right)$

Vapor phase

$T I N_{s^{\prime}, k}^{H} \leq \beta_{s^{\prime}}^{U} U_{s}^{V P}$

$T I N_{s^{\prime}, k}^{C} \leq \beta_{s^{\prime}}^{U} V_{S}^{V P}$

$\operatorname{TOUT}_{s^{\prime}, k}^{H} \leq \gamma_{S^{\prime}}^{U}\left(U_{S}^{V P}+V_{s}^{V P}\right)$

$\operatorname{TOUT}_{s^{\prime}, k}^{C} \leq \gamma_{S^{\prime}}^{U} V_{s}^{V P}$

$\sum_{k} \operatorname{TOUT}_{s^{\prime}, k}^{H} \leq T_{s}^{D E W}+\gamma_{s^{\prime}}^{U}\left(1-U_{s}^{V P}+V_{s}^{V P}\right)$

$\sum_{k}$ TOUT $_{s^{\prime}, k}^{H} \geq T_{S}^{D E W}-\gamma_{s^{\prime}}^{U}\left(1-U_{S}^{V P}+V_{s}^{V P}\right)$

$\sum_{k} T I N_{s^{\prime}, k}^{C} \leq T_{S}^{D E W}+\beta_{s^{\prime}}^{U}\left(1-V_{s}^{V P}+U_{S}^{V P}\right)$

$\sum_{k} T I N_{s^{\prime}, k}^{C} \geq T_{s}^{D E W}-\beta_{s^{\prime}}^{U}\left(1-V_{s}^{V P}+U_{s}^{V P}\right)$ $s \in \mathbf{S}^{\mathbf{P C}}, s^{\prime} \in \mathbf{S}_{s}^{\mathbf{2 P}}, k \in \mathbf{K}$

$s \in \mathbf{S}^{\mathbf{P C}}, s^{\prime} \in \mathbf{S}_{s}^{\mathbf{2 P}}, k \in \mathbf{K}^{\mathbf{I}}$

$s \in \mathbf{S}^{\mathbf{P C}}, s^{\prime} \in \mathbf{S}_{s}^{\mathbf{P}}, k \in \mathbf{K}^{\mathbf{I}}$

$s \in \mathbf{S}^{\mathbf{P C}}, s^{\prime} \in \mathbf{S}_{s}^{2 \mathbf{P}}, k \in \mathbf{K}$

$s \in \mathbf{S}^{\mathbf{P C}}, s^{\prime} \in \mathbf{S}_{S}^{2 \mathbf{P}}, k \in \mathbf{K}$

$s \in \mathbf{S}^{\mathbf{P C}}, s^{\prime} \in \mathbf{S}_{S}^{\mathbf{2 P}}, k \in \mathbf{K}^{\mathbf{I}}$

$s \in \mathbf{S}^{\mathbf{P C}}, s^{\prime} \in \mathbf{S}_{S}^{\mathbf{2 P}}, k \in \mathbf{K}^{\mathbf{I}}$

$s \in \mathbf{S}^{\mathbf{P C}}, s^{\prime} \in \mathbf{S}_{s}^{\mathbf{2 P}}$

$s \in \mathbf{S}^{\mathbf{P C}}, s^{\prime} \in \mathbf{S}_{S}^{\mathbf{2 P}}$

$s \in \mathbf{S}^{\mathbf{P C}}, s^{\prime} \in \mathbf{S}_{s}^{2 \mathbf{P}}$

$s \in \mathbf{S}^{\mathbf{P C}}, s^{\prime} \in \mathbf{S}_{s}^{2 \mathbf{P}}$

$s \in \mathbf{S}^{\mathbf{P C}}, s^{\prime} \in \mathbf{S}_{s}^{\mathbf{2 P}}$

$s \in \mathbf{S}^{\mathbf{P C}}, s^{\prime} \in \mathbf{S}_{S}^{2 \mathbf{P}}$

$s \in \mathbf{S}^{\mathbf{P C}}, s^{\prime} \in \mathbf{S}_{s}^{2 \mathbf{P}}$

$s \in \mathbf{S}^{\mathbf{P C}}, s^{\prime} \in \mathbf{S}_{s}^{\mathbf{2 P}}$

$s \in \mathbf{S}^{\mathbf{P C}}, s^{\prime} \in \mathbf{S}_{S}^{2 \mathbf{P}}$

$s \in \mathbf{S}^{\mathbf{P C}}, s^{\prime} \in \mathbf{S}_{s}^{2 \mathbf{P}}$

$s \in \mathbf{S}^{\mathbf{P C}}, s^{\prime} \in \mathbf{S}_{S}^{\mathbf{2 P}}$

$s \in \mathbf{S}^{\mathbf{P C}}, s^{\prime} \in \mathbf{S}_{s}^{2 \mathbf{P}}$

(A18)

$s \in \mathbf{S}^{\mathbf{P C}}, s^{\prime} \in \mathbf{S}_{s}^{\mathrm{VP}}, k \in \mathbf{K}$

$s \in \mathbf{S}^{\mathbf{P C}}, s^{\prime} \in \mathbf{S}_{s}^{\mathbf{V P}}, k \in \mathbf{K}$

$s \in \mathbf{S}^{\mathbf{P C}}, s^{\prime} \in \mathbf{S}_{s}^{\mathbf{V P}}, k \in \mathbf{K}^{\mathbf{I}}$

$s \in \mathbf{S}^{\mathbf{P C}}, s^{\prime} \in \mathbf{S}_{s}^{\mathbf{V P}}, k \in \mathbf{K}^{\mathbf{I}}$

$s \in \mathbf{S}^{\mathbf{P C}}, s^{\prime} \in \mathbf{S}_{s}^{\mathbf{V P}}$

$s \in \mathbf{S}^{\mathbf{P C}}, s^{\prime} \in \mathbf{S}_{s}^{\mathrm{VP}}$

$s \in \mathbf{S}^{\mathbf{P C}}, s^{\prime} \in \mathbf{S}_{s}^{\mathbf{V P}}$

$s \in \mathbf{S}^{\mathbf{P C}}, s^{\prime} \in \mathbf{S}_{s}^{\mathbf{V P}}$ 
$\sum_{k}\left(T O U T_{s^{\prime}, k}^{H}+\operatorname{TIN}_{s^{\prime}, k}^{C}\right) \geq T_{s}^{D E W}\left(V_{s}^{V P}+U_{s}^{V P}-1\right) \quad s \in \mathbf{S}^{\mathbf{P C}}, s^{\prime} \in \mathbf{S}_{s}^{\mathrm{VP}}$

\section{A.3 Example}

To illustrate let us consider an example that represents Case 2 in Table 1: stream $s$ starts as liquid and ends in the two-phase region. Therefore, $U_{s}^{L Q}=U_{s^{\prime} \in \mathbf{S}_{s}^{\mathbf{L Q}}}=1$ and $V_{s}^{2 P}=V_{s^{\prime} \in \mathbf{S}_{s}^{2 P}}=1$, while other binaries $U$ and $V$ are all equal to zero due to Eqs. (34) and (35). Then, Eqs. (36) - (43) or (A1) - (A8) ensure that the parent stream inlet temperature matches the liquid sub-stream inlet temperature, and the parent stream outlet temperature matches the two-phase sub-stream outlet temperature.

Since both parent stream and sub-streams in Case 2 are cold streams, all $T_{I} N_{s^{\prime}, k}^{H}$ and $T O U T_{s^{\prime}, k}^{H}$ are forced to zero by Eqs. $(10 \mathrm{H})$ and $(15 \mathrm{H})$, respectively. Therefore, constraints that "control" these variables (e.g. Eqs. (A37) and (A39)) become redundant. The only "active" constraints in this example are as follows:

1. Eqs. (A15) and (A16), which match the outlet temperature of the liquid sub-stream with the bubble point.

2. Eqs. (A31) and (A32), which match the inlet temperature of the two-phase sub-stream with the bubble point, and

3. Eqs. (A38) and (A40), which force both inlet and outlet temperatures of the vapor sub-stream to zero. 


\section{Appendix B. Preprocessing Algorithms}

Algorithm B1. Preprocessing for a preclassified hot stream $\left(s \in \mathbf{S}^{\mathbf{H}}\right)$

1: if $s \in \mathbf{S}^{\mathbf{H}}$ then

2: $\quad W_{s}^{C}=0, W_{s}^{H}=1, T_{s}^{-}=0$

$\left.Q_{s, k}^{C}=Q 1_{s, k}^{C}=Q 1_{s, k}^{C, D}=Q 2_{s, k}^{C}=Q 2_{s, k}^{C, D}=Q 2_{s, k}^{C, P}=0\right)$

3:

$$
\begin{aligned}
& \operatorname{TIN}_{s, k}^{C}=\operatorname{TOUT}_{s, k}^{C}=0 \\
& X_{s, k}^{C}=Y_{s, k}^{C}=Z_{s, k}^{C}=0
\end{aligned}
$$

4: $\quad$ remove constraints with suffix " $C$ ", and replace $W_{s}^{H}$ with 1 in Eqs. (3), (7H), and (13H).

5: $\quad$ for $k^{\prime} \in \mathbf{K}$

6: $\quad$ if $T I N_{S}$ is the $k^{\prime}$ th highest, then

7:

8:

9:

10:

11:

12:

13:

14:

$15:$

16:

17:

18:

19:

20:

21:

22:

23:

24:

25

26:

$$
\operatorname{TIN}_{s}=T_{k^{\prime}}, X_{s, k^{\prime}}^{H}=1 \text {, and }
$$$$
X_{s, k}^{H}=\operatorname{TIN}_{s, k}^{H}=0 \quad \forall k \neq k^{\prime}
$$

$$
\left.\begin{array}{c}
Y_{s, k}^{H}=\operatorname{TOUT}_{s, k}^{H}=Q 2_{s, k}^{H}=0 \\
Z_{s, k}^{H}=Q 1_{s, k}^{H}=0
\end{array}\right\}
$$$$
\forall k \leq k^{\prime}
$$

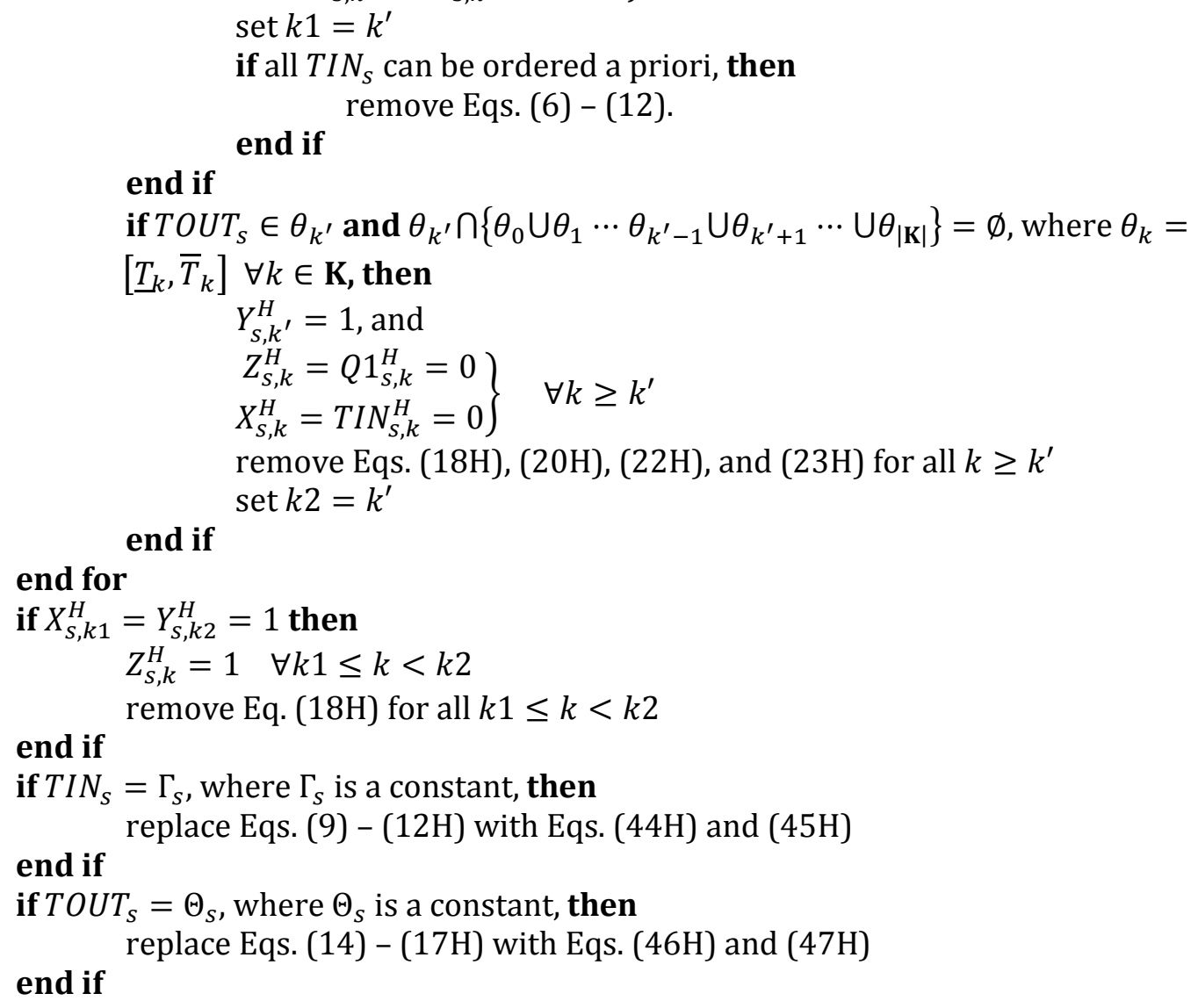

$$
\text { set } k 1=k^{\prime}
$$


Algorithm B2. Preprocessing for a preclassified cold stream $\left(s \in \mathbf{S}^{\mathbf{C}}\right)$

\section{1 : if $s \in \mathbf{S}^{\mathbf{C}}$ then}

2: $\quad W_{s}^{H}=0, W_{s}^{C}=1, T_{s}^{+}=0$

$$
\left.\begin{array}{c}
Q_{s, k}^{H}=Q 1_{s, k}^{H}=Q 1_{s, k}^{H, D}=Q 2_{s, k}^{H}=Q 2_{s, k}^{H, D}=Q 2_{s, k}^{H, P}=0 \\
T I N_{s, k}^{H}=T O U T_{s, k}^{H}=0 \\
X_{s, k}^{H}=Y_{s, k}^{H}=Z_{s, k}^{H}=0
\end{array}\right\} \forall k \in \mathbf{K}
$$

3:

4: $\quad$ remove constraints with suffix " $\mathrm{H}$ ", and replace $W_{s}^{C}$ with 1 in Eqs. (4), (7C), and (13C).

5: $\quad$ for $k^{\prime} \in \mathbf{K}$

6: $\quad$ if $T I N_{S}$ is the $k^{\prime}$ th highest, then

7:

8:

9:

10:

11:

12:

13:

14:

15:

16:

17:

18:

19:

20:

21:

22:

23:

24:

25:

26:

27:

28:

29:

30:

31:

32:

$$
\begin{aligned}
& T I N_{s}=T_{k^{\prime}}, X_{s, k^{\prime}}^{C}=1, \text { and } \\
& X_{s, k}^{C}=T I N_{s, k}^{C}=0
\end{aligned} \forall k \neq k^{\prime}
$$$$
\left.\begin{array}{c}
Y_{s, k}^{C}=\operatorname{TOUT}_{s, k}^{C}=Q 2_{s, k}^{C}=0 \\
Z_{s, k}^{C}=Q 1_{s, k}^{C}=0
\end{array}\right\} \forall k>k^{\prime}
$$

set $k 1=k^{\prime}$

if all $T I N_{S}$ can be ordered a priori, then

end if

$$
\text { end if }
$$$$
\text { remove Eqs. (6) - (12). }
$$

if $\operatorname{TOUT}_{s} \in \theta_{k^{\prime}}$ and $\theta_{k^{\prime}} \cap\left\{\theta_{0} \cup \theta_{1} \cdots \theta_{k^{\prime}-1} \cup \theta_{k^{\prime}+1} \cdots \cup \theta_{|\mathbf{K}|}\right\}=\emptyset$, where $\theta_{k}=$ $\left[\underline{T}_{k}, \bar{T}_{k}\right] \forall k \in \mathbf{K}$, then

$Y_{s, k^{\prime}}^{C}=1$, and

$Z_{s, k}^{C}=Q 1_{s, k}^{C}=0$

$\left.X_{s, k}^{C}=\operatorname{TIN}_{s, k}^{C}=0\right\}$

$\forall k<k^{\prime}$

remove Eqs. (18C), (20C), (22C), and (23C) for all $k<k^{\prime}$

end if

end for

if $X_{s, k 1}^{C}=Y_{s, k 2}^{C}=1$ then

$$
Z_{s, k}^{C}=1 \quad \forall k 2 \leq k<k 1
$$

end if

remove Eq. (18C) for all $k 2 \leq k<k 1$

if $\operatorname{TIN}_{S}=\Gamma_{S}$, where $\Gamma_{S}$ is a constant, then

end if

replace Eqs. (9) - (12C) with Eqs. (44C) and (45C)

if $\operatorname{TOUT}_{s}=\Theta_{s}$, where $\Theta_{s}$ is a constant, then

end if

replace Eqs. (14) - (17C) with Eqs. (46C) and (47C)

33: end if 
Algorithm B3. Preprocessing for an unclassified stream $\left(s \in \mathbf{S}^{\mathbf{U N}}\right)$

$1:$ if $s \in \mathbf{S}^{\mathbf{U N}}$ then

2: $\quad$ for $k^{\prime} \in \mathbf{K}$

3: $\quad$ if $\operatorname{TIN}_{S}$ is the $k^{\prime}$ th highest, then

4: $\quad \operatorname{TIN}_{s}=T_{k^{\prime}}$

5:

6:

$$
\left.\begin{array}{l}
X_{s, k}^{H}=T I N_{s, k}^{H}=0 \\
X_{s, k}^{C}=T I N_{s, k}^{C}=0
\end{array}\right\}
$$$$
\forall k \neq k^{\prime}
$$

$\left.Y_{s, k}^{H}=\operatorname{TOUT}_{s, k}^{H}=Q 2_{s, k}^{H}=0\right\}$

$$
\left.\begin{array}{l}
Z_{s, k}^{H}=Q 1_{s, k}^{H}=0
\end{array}\right\} \quad \forall k \leq k^{\prime}
$$

7:

$$
\left.\begin{array}{c}
Y_{s, k}^{C}=\operatorname{TOUT}_{s, k}^{C}=Q 2_{s, k}^{C}=0 \\
Z_{s, k}^{C}=Q 1_{s, k}^{C}=0
\end{array}\right\} \quad \forall k>k^{\prime}
$$

set $k 1=k^{\prime}$

8:

if all $\operatorname{TIN}_{S}$ can be ordered a priori, then

10:

11:

12:

13:

14:

15:

\section{end if}

\section{end if} remove Eqs. (6) - (12).

if $\operatorname{TOUT}_{s} \in \theta_{k^{\prime}}$ and $\theta_{k^{\prime}} \cap\left\{\theta_{0} \cup \theta_{1} \cdots \theta_{k^{\prime}-1} \cup \theta_{k^{\prime}+1} \cdots \cup \theta_{|\mathbf{K}|}\right\}=\emptyset$, where $\theta_{k}=$ $\left[\underline{T}_{k}, \bar{T}_{k}\right] \forall k \in \mathbf{K}$, then

$$
\left.\begin{array}{c}
Z_{s, k}^{H}=Q 1_{s, k}^{H}=0 \\
X_{s, k}^{H}=T I N_{s, k}^{H}=0
\end{array}\right\} \quad \forall k \geq k^{\prime}
$$

16:

$$
\left.Z_{s, k}^{C}=Q 1_{s, k}^{C}=0\right)
$$$$
\left.X_{s, k}^{C}=T I N_{s, k}^{C}=0\right\}
$$$$
\forall k<k^{\prime}
$$

17:

18:

19:

20:

21:

22:

23:

24:

25:

26:

27:

28:

29:

$30:$ remove Eqs. (18H), (20H), (22H), and (23H) for all $k \geq k^{\prime}$ remove Eqs. (18C), (20C), (22C), and (23C) for all $k<k^{\prime}$

\section{end if}

\section{end for}

$$
\text { if } X_{s, k 1}^{H}=Y_{s, k 2}^{H}=1 \text { then }
$$

if $k 1 \leq k 2$ then

$$
Z_{s, k}^{H}=1 \quad \forall k 1 \leq k<k 2
$$

else if $k 1>k 2$ then

$$
\text { remove Eq. (18H) for all } k 1 \leq k<k 2
$$

$$
Z_{s, k}^{C}=1 \quad \forall k 2 \leq k<k 1
$$

remove Eq. (18C) for all $k 2 \leq k<k 1$

\section{end if}

\section{end if}

31: if TIN $_{S}=\Gamma_{S}$, where $\Gamma_{S}$ is a constant, then

32:

33:

$$
\text { replace Eqs. (9) - (12C) with Eqs. (44H) - (45C) }
$$

34: if TOUT $_{s}=\Theta_{s}$, where $\Theta_{s}$ is a constant, then

35: $\quad$ replace Eqs. (14) - (17C) with Eqs. $(46 \mathrm{H})-(47 \mathrm{C})$

36: $\quad$ end if 


\section{Appendix C. Additional Information for Example in \$7.2}

RXN $3(C \rightleftharpoons D)$ is assumed to be an equilibrium reaction. The equilibrium constant $\left(K_{c}\right)$ is a function of the temperature of the reactor (CSTR3):

$\ln K_{C}=\ln K_{C}^{0}-\frac{\Delta H^{0}}{\mathcal{R}}\left(\frac{1}{T}-\frac{1}{298}\right)$

where $\Delta H^{0}=8 \mathrm{~kJ} / \mathrm{mol}$ is the heat of reaction at standard state $(298 \mathrm{~K}, 1 \mathrm{bar}), K_{c}^{0}=0.4$ is the equilibrium constant at standard state, $\mathcal{R}$ is the universal gas constant, and $T$ is the temperature of CSTR3.

For simplicity, we assume ideal behavior:

$K_{C}=\frac{[D]}{[C]}$

where $[C]$ and $[D]$ are the concentration of component $C$ and D in stream 13 , respectively.

Table C1. Optimal conditions for streams in the process $(s \in \mathbb{S})$

\begin{tabular}{c|cccc|c}
\hline \multirow{2}{*}{ Stream \# } & \multicolumn{3}{|c|}{ Component flow (kmol/s) } & Temperature \\
\cline { 2 - 4 } & $\mathrm{A}$ & $\mathrm{B}$ & $\mathrm{C}$ & $\mathrm{D}$ & $(\mathrm{K})$ \\
\hline 1 & 2.00 & 1.00 & & & 300 \\
$2^{*}$ & 2.23 & 1.02 & & & $310 \rightarrow 500$ \\
3 & 2.23 & 1.02 & & & 500 \\
5 & 1.32 & 0.10 & 0.92 & & 500 \\
$7^{*}$ & 1.32 & 0.10 & 0.92 & & $500 \rightarrow 430$ \\
8 & 1.32 & 0.10 & & & 430 \\
9 & 1.08 & 0.08 & & & 430 \\
10 & 0.23 & 0.02 & & & 430 \\
11 & & & 0.93 & & 430 \\
$12^{*}$ & & & 2.01 & & $386 \rightarrow 386$ \\
$13^{*}$ & & & 1.09 & 0.92 & $386 \rightarrow 350$ \\
$15^{*}$ & & & & & $380 \rightarrow 340$ \\
22 & & & 1.09 & 0.92 & 350 \\
23 & & & & 0.92 & 350 \\
24 & & & 1.09 & & 350 \\
26 & & & 1.09 & & 350 \\
27 & & & & 0.92 & 350 \\
\hline
\end{tabular}

Note that streams with "*” require heating/cooling, and thus their temperatures are changed according to the heating/cooling requirements. Streams not shown have zero flow rates. 
Table C2. Optimal conditions for streams in the heat integration $\left(s \in \mathbf{S}^{\mathbf{P}}\right)$

\begin{tabular}{ccccc}
\hline Stream \# & Class & TIN (K) & TOUT (K) & $\begin{array}{c}\text { Heat flow } \\
(\mathrm{kW} / \mathrm{K})\end{array}$ \\
\hline 2 & Cold & 310.1 & 500 & 65.0 \\
7 & Hot & 500 & 430 & 55.9 \\
12 & - & 386.5 & 386.5 & 60.3 \\
13 & Hot & 386.5 & 350 & 64.9 \\
15 & Hot & 380 & 340 & 0 \\
\hline Isothermal & & & & Heat duty \\
streams & & & $\mathrm{kW})$ \\
\hline SEP1 & Hot & 386.5 & - & 720 \\
CSTR3 & Hot & 430 & - & 7330 \\
\hline
\end{tabular}




\section{Notation}

\section{Sets}

$i \in \mathbf{I}$

processing units

$s \in \mathbf{S}$

$s \in \mathbb{S}$

streams in the heat integration model

$k \in \mathbf{K}$

streams in the process model

temperature interval boundaries

$\begin{array}{ll}\begin{array}{l}\text { Subsets } \\ \mathbf{S}^{\mathbf{H U}}\end{array} & \text { hot utility streams } \\ \mathbf{S}^{\mathbf{H}} & \text { preclassified hot streams } \\ \mathbf{S}^{\mathbf{C U}} & \text { cold utility streams } \\ \mathbf{S}^{\mathbf{C}} & \text { preclassified cold streams } \\ \mathbf{S}^{\mathbf{I H U}} & \text { isothermal hot utility streams } \\ \mathbf{S}^{\mathbf{I C U}} & \text { isothermal cold utility streams } \\ \mathbf{S}^{\mathbf{I P}} & \text { isothermal process streams } \\ \mathbf{S}^{\mathbf{P}} & \text { process streams that requires heating/cooling } \\ \mathbf{S}^{\mathbf{P C}} & \text { process streams with potential phase changes } \\ \mathbf{S}_{S}^{\mathbf{S U B}} & \text { sub-streams associated with parent stream s } \\ \mathbf{S}^{\mathbf{U N}} & \text { unclassified streams } \\ \mathbf{K}^{\mathbf{I}} & \text { temperature intervals }\end{array}$

Parameters

$\alpha_{S}^{U}$

upper bound on inlet and outlet temperature difference

$\beta_{S}^{U}$

$\gamma_{S}^{U}$

$\delta$

$\theta_{S}^{U}$

$\lambda_{s}$

$\mu_{S}$

$T_{S}^{B B L}$

upper bound on inlet temperature of stream $s$

upper bound on outlet temperature of stream $s$

heat recovery approach temperature/minimum approach temperature

upper bound on heat duty of stream $s$

latent heat of stream $s$

unit cost of utility stream $s$

bubble point of parent stream $s$

$T_{S}^{D E W}$

dew point of parent stream $s$

\section{Non-negative Continuous Variables}

$\Psi$

a vector of variables in the chemical process model 


$\begin{array}{ll}F_{S} & \text { heat capacity flowrate of process stream } s \\ \widehat{F}_{S} & \text { mass flowrate of process stream } s \\ K_{C} & \text { reaction equilibrium constant } \\ P_{i} & \text { pressure of unit } i \\ Q_{i} & \text { heat duty of unit } i \\ Q_{S, k}^{H} / Q_{S, k}^{C} & \text { true heat duty of stream } s \text { in interval } k \\ Q 1_{s, k}^{H} / Q 1_{s, k}^{C} & \text { disaggregated heat duty in case (1) } \\ Q 1_{s, k}^{H, D} / Q 1_{s, k}^{C, D} & \text { dummy disaggregated heat duty in case (1) } \\ Q 2_{s, k}^{H} / Q 2_{s, k}^{C} & \text { disaggregated heat duty in case (2) } \\ Q 2_{s, k}^{C, P} & \text { dummy disaggregated heat duty in case (2) } \\ Q 2_{s, k}^{H, D} / Q 2_{s, k}^{C, D} & \text { dummy disaggregated heat duty in case (2) } \\ R_{k} & \text { residual heat at interval } k \\ T_{k} & \text { temperature of interval boundary } k \\ T I N_{S} & \text { inlet temperature of stream } s \\ T I N_{S^{\prime}}^{P} & \text { disaggregated inlet temperature of sub-stream } s^{\prime} \\ T I N_{S, k}^{H} / T I N_{S, k}^{C} & \text { disaggregated inlet temperature of stream } s \text { at interval boundary } k \\ T O U T_{S} & \text { outlet temperature of stream } s \\ T O U T_{s^{\prime}}^{P} & \text { disaggregated outlet temperature of sub-stream } s^{\prime} \\ T O U T_{S, k}^{H} / T O U T_{S, k}^{C} & \text { disaggregated outlet temperature of stream } s \text { at interval } k \\ T_{S}^{+} / T_{S}^{-} & \text {deviation variable for temperature differences } \\ W_{i} & \end{array}$

\section{Binary Variables}

$U_{s}$

$V_{S}$

$W_{S}^{H} / W_{S}^{C}$

$X_{s, k}^{H} / X_{s, k}^{C}$

$Y_{S, k}^{H} / Y_{s, k}^{C}$

$Y_{i}^{U N I T}$

$Z_{s, k}^{H} / Z_{s, k}^{C}$

equal to 1 if sub-stream $s$ is the starting stream

equal to 1 if sub-stream $\mathrm{s}$ is the ending stream

equal to 1 if stream $s$ is a hot/cold stream

equal to 1 if stream $s$ starts at boundary $k$

equal to 1 if stream $s$ ends at interval $k$

equal to 1 if processing unit $i$ is selected in the superstructure

equal to 1 if stream $s$ goes through interval $k$ 


\section{References}

Balakrishna, S., \& Biegler, L. T. (1992). Targeting strategies for the synthesis and energy integration of nonisothermal reactor networks. Industrial \& engineering chemistry research, 31(9), 21522164. doi:10.1021/ie00009a013

Bausa, J., Von Watzdorf, R., \& Marquardt, W. (1998). Shortcut methods for nonideal multicomponent distillation: 1. Simple columns. Aiche Journal, 44(10), 2181-2198. doi:DOI 10.1002/aic.690441008

Boukouvala, F., \& Ierapetritou, M. G. (2013). Surrogate-based optimization of expensive flowsheet modeling for continuous pharmaceutical manufacturing. Journal of Pharmaceutical Innovation, 8(2), 131-145.

Cerda, J., Westerberg, A. W., Mason, D., \& Linnhoff, B. (1983). Minimum utility usage in heat exchanger network synthesis A transportation problem. Chemical Engineering Science, 38(3), 373-387. doi:Doi: 10.1016/0009-2509(83)80156-0

Ciric, A. R., \& Floudas, C. A. (1991). Heat exchanger network synthesis without decomposition. Computers \& chemical engineering, 15(6), 385-396. doi:Doi: 10.1016/0098-1354(91)870174

Dowling, A. W., \& Biegler, L. T. (2015). A framework for efficient large scale equation-oriented flowsheet optimization. Computers \& chemical engineering, 72, 3-20.

Duran, M. A., \& Grossmann, I. E. (1986). Simultaneous optimization and heat integration of chemical processes. AIChE Journal, 32(1), 123-138. doi:10.1002/aic.690320114

El - Halwagi, M. M., \& Manousiouthakis, V. (1990). Simultaneous synthesis of mass - exchange and regeneration networks. AIChE Journal, 36(8), 1209-1219.

Furman, K. C., \& Sahinidis, N. V. (2001). Computational complexity of heat exchanger network synthesis. Computers \& chemical engineering, 25(9-10), 1371-1390. doi:Doi: 10.1016/s00981354(01)00681-0

Gani, R., \& Bek - Pedersen, E. (2000). Simple new algorithm for distillation column design. AIChE Journal, 46(6), 1271-1274.

Grossmann, I. E., Yeomans, H., \& Kravanja, Z. (1998). A rigorous disjunctive optimization model for simultaneous flowsheet optimization and heat integration. Computers \& chemical engineering, 22, Supplement 1, S157-S164. doi:http://dx.doi.org/10.1016/S0098-1354(98)00050-7

Gundersen, T., \& Naess, L. (1988). The synthesis of cost optimal heat exchanger networks: An industrial review of the state of the art. Computers \& chemical engineering, 12(6), 503-530. doi:10.1016/0098-1354(88)87002-9

Hasan, M., Jayaraman, G., Karimi, I., \& Alfadala, H. (2010). Synthesis of heat exchanger networks with nonisothermal phase changes. AIChE Journal, 56(4), 930-945.

Hohmann, E. C. (1971). Optimum networks for heat exchange. (PhD), University of Southern California, Los Angeles, CA

Hui, C.-W. (2014). Optimization of heat integration with variable stream data and non-linear process constraints. Computers \& chemical engineering, 65, 81-88. doi:http://dx.doi.org/10.1016/i.compchemeng.2014.03.010

Jeżowski, J. (1994). Heat exchanger network grassroot and retrofit design. The review of the stateof-the-art: Part I. Heat exchanger network targeting and insight based methods of synthesis. Hungarian Journal of Industrial Chemistry, 22(4), 279-294.

Kamath, R. S., Biegler, L. T., \& Grossmann, I. E. (2012). Modeling multistream heat exchangers with and without phase changes for simultaneous optimization and heat integration. AIChE Journal, 58(1), 190-204. 
Klemeš, J. J., \& Kravanja, Z. (2013). Forty years of Heat Integration: Pinch Analysis (PA) and Mathematical Programming (MP). Current Opinion in Chemical Engineering, 2(4), 461-474. doi:http://dx.doi.org/10.1016/j.coche.2013.10.003

Kong, L., Sen, S. M., Henao, C. A., Dumesic, J. A., \& Maravelias, C. T. (2016). A superstructure-based framework for simultaneous process synthesis, heat integration, and utility plant design. Computers \& chemical engineering.

Lee, S., \& Grossmann, I. E. (2005). Logic-based modeling and solution of nonlinear discrete/continuous optimization problems. Annals of Operations Research, 139(1), 267-288.

Linnhoff, B., \& Flower, J. R. (1978). Synthesis of heat exchanger networks: I. Systematic generation of energy optimal networks. AIChE Journal, 24(4), 633-642. doi:10.1002/aic.690240411

Linnhoff, B., \& Hindmarsh, E. (1983). The pinch design method for heat exchanger networks. Chemical Engineering Science, 38(5), 745-763. doi:Doi: 10.1016/0009-2509(83)80185-7

Morar, M., \& Agachi, P. S. (2010). Review: Important contributions in development and improvement of the heat integration techniques. Computers \& chemical engineering, 34(8), 1171-1179. doi:http://dx.doi.org/10.1016/j.compchemeng.2010.02.038

Navarro-Amorós, M. A., Caballero, J. A., Ruiz-Femenia, R., \& Grossmann, I. E. (2013). An alternative disjunctive optimization model for heat integration with variable temperatures. Computers \& chemical engineering, 56, 12-26.

Papoulias, S. A., \& Grossmann, I. E. (1983). A structural optimization approach in process synthesisI. Computers \& chemical engineering, 7(6), 695-706. doi:http://dx.doi.org/10.1016/00981354(83)85022-4

Pattison, R. C., \& Baldea, M. (2015). Multistream heat exchangers: Equation - oriented modeling and flowsheet optimization. AIChE Journal, 61(6), 1856-1866.

Ponce-Ortega, J. M., Jiménez-Gutiérrez, A., \& Grossmann, I. E. (2008). Optimal synthesis of heat exchanger networks involving isothermal process streams. Computers \& chemical engineering, 32(8), 1918-1942.

Srinivas, B., \& El-Halwagi, M. (1994). Synthesis of combined heat and reactive mass-exchange networks. Chemical Engineering Science, 49(13), 2059-2074.

Tan, Y. L., Ng, D. K. S., El-Halwagi, M. M., Foo, D. C. Y., \& Samyudia, Y. (2014). Floating pinch method for utility targeting in heat exchanger network (HEN). Chemical Engineering Research and Design, 92(1), 119-126. doi:http://dx.doi.org/10.1016/j.cherd.2013.06.029

Tawarmalani, M., \& Sahinidis, N. V. (2005). A polyhedral branch-and-cut approach to global optimization. Mathematical Programming, 103(2), 225-249. doi:10.1007/s10107-005-05818

Türkay, M., \& Grossmann, I. E. (1996). Disjunctive programming techniques for the optimization of process systems with discontinuous investment costs-multiple size regions. Industrial \& engineering chemistry research, 35(8), 2611-2623.

Wu, W., Henao, C. A., \& Maravelias, C. T. (2016). A Superstructure Representation, Generation and Modeling Framework for Chemical Process Synthesis. AIChE Journal.

Yee, T. F., \& Grossmann, I. E. (1990). Simultaneous optimization models for heat integration--II. Heat exchanger network synthesis. Computers \& chemical engineering, 14(10), 1165-1184. doi:Doi: 10.1016/0098-1354(90)85010-8

Yuan, Z., \& Eden, M. R. (2016). Superstructure optimization of integrated fast pyrolysis - gasification for production of liquid fuels and propylene. AIChE Journal. 
Heat integration

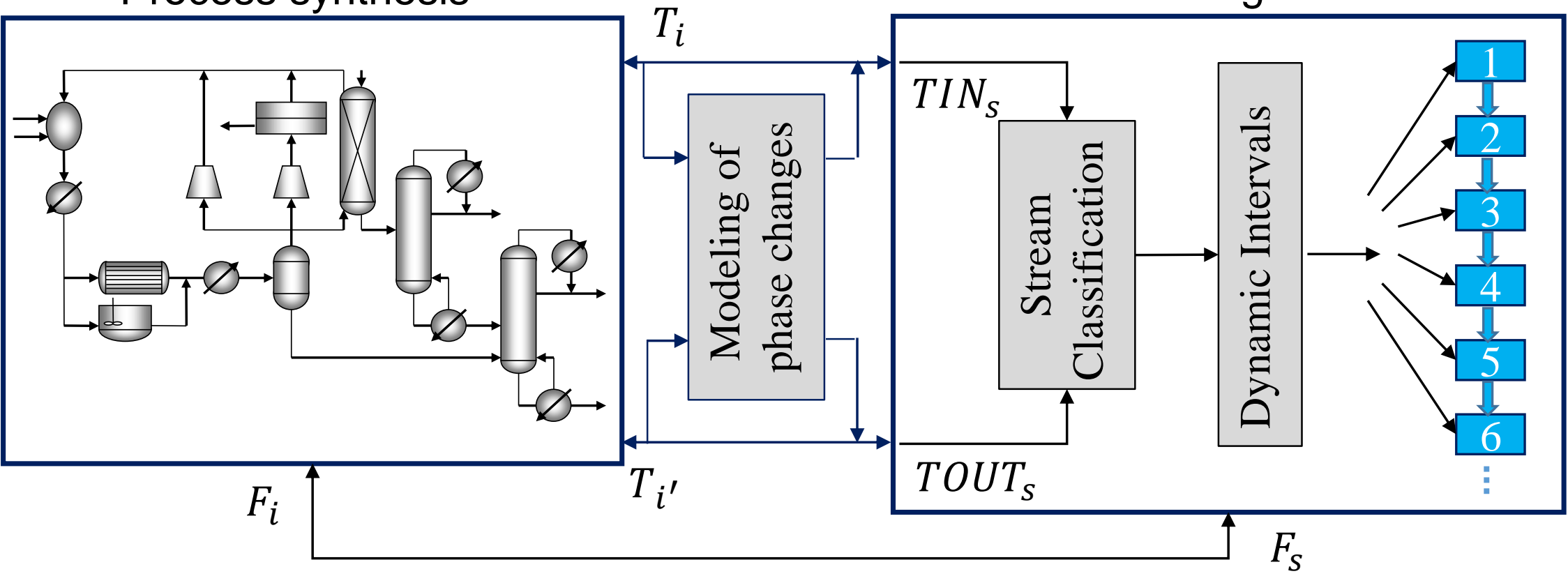

